Aus der Abteilung Neuroradiologie

(Prof. Dr. med. M. Knauth)

im Zentrum Radiologie

der Medizinischen Fakultät der Universität Göttingen

\title{
Der Einfluss von Plaquemorphologie und anderen prädiktiven Faktoren auf das Auftreten von Mikroembolien während der Stentgeschützten Perkutanen Angioplastie der Karotiden (SPAC)
}

\author{
INAUGURAL- DISSERTATION \\ zur Erlangung des Doktorgrades \\ der Medizinischen Fakultät \\ der Georg-August-Universität zu Göttingen
}

vorgelegt von

Peter Weber

aus

Pfaffenhofen an der IIm 


\section{INHALTSVERZEICHNIS}

1. EINLEITUNG 3

2. GRUNDLAGEN 5

2.1. Schlaganfall und TIA 5

2.1.1. Epidemiologische Daten 5

2.1.2. Einteilung und Klinik 5

2.2. Karotisstenose 6

2.2.1. Ätiologie und Pathogenese 6

2.2.2. Einflussfaktoren auf die Plaquemorphologie 8

2.2.3. Bildgebende Diagnostik 9

2.2.3.1. Sonographie 9

2.2.3.2. CT-Angiographie 10

2.2.3.3. MR-Angiographie 11

2.2.3.4. Digitale Subtraktionsangiographie 11

$\begin{array}{lr}\text { 2.2.4. Therapie } & 12\end{array}$

2.2.4.1. Karotisendarterektomie 12

2.2.4.2. Stentgeschützte Perkutane Angioplastie der Karotiden 13

2.2.4.3. Zerebrale Protektionssysteme 14

3. FRAGESTELLUNG 16

$\begin{array}{ll}\text { 4. METHODE } & 18\end{array}$

4.1. Studiendesign und Patientenkollektiv 18

$\begin{array}{ll}\text { 4.2. SPAC } & 19\end{array}$

4.2.1. Entlüftung und Durchspülung des Stentsystems 19

4.3. Plaquecharakterisierung in der CTA 20

4.3.1. Prinzip der CTA 20

4.3.2. CTA-Parameter 22

4.3.3. Software 22

4.3.4. Bestimmung des Stenosegrades 22

4.3.5. Messung von Weich- und Hartplaque 23

4.4. Klassifizierung der Plaquemorphologie nach Lovett et al. 25

4.4.1. Lovett- Klassifikation der DSA 25

4.4.2. Lovett- Klassifikation der CTA 25 
4.5.1. Grundlagen der kraniellen MRT 26

4.5.1.1. Diffusionsgewichtete Bildgebung 27

4.5.1.2. b-Wert und Infarktdarstellung 28

4.5.2. MRT-Parameter 28

4.5.3. Software 29

4.5.4. Messung der DWI-Läsionen 29

4.6. Statistische Auswertung 31

5. ERGEBNISSE

5.1. Deskriptive Statistik 32

5.2. Zusammenhang Plaquezusammensetzung und DWI-Läsionen 34

5.3. Zusammenhang Ulzerationen und DWI-Läsionen 37

5.4. Andere Risikofaktoren anhand der CTA 38

5.5. Klassifikation der Plaquemorphologie nach Lovett et al. 39

5.6. Einsatz von zerebralen Protektionssystemen 43

5.7. Technische Details der SPAC 44

6. DISKUSSION 46

6.1. SPAC 46

6.2. Zerebrale Protektionssysteme 47

6.3. CTA und DWI-MRT 49

6.4. Zusammenhang Plaquemorphologie und DWI-Läsionen 50

6.5. Ulzerationen und Lovettklassifikation als Risikofaktoren 51

6.6. Andere Risikofaktoren 53

7. ZUSAMMENFASSUNG 55

8. LITERATURVERZEICHNIS 


\section{Einleitung}

In unserer Gesellschaft spielen kardio- und zerebrovaskuläre Erkrankungen, bedingt durch eine steigende Lebenserwartung, eine zunehmend bedeutende Rolle. Bluthochdruck und Atherosklerose stehen als beeinflussbare Faktoren der Gefäßwandschädigung im Fokus der medizinischen Forschung. Stenosen der hirnzuführenden Gefäße, wie die Karotisstenose, haben ohne ausreichende Kollateralisation zum einen eine Minderperfusion des Gehirns zur Folge, zum anderen ist die sie verursachende Plaque thrombogen und kann durch ihre Embolien zu einem Gefäßverschluss im Gehirn und somit zum Schlaganfall führen. Der Schlaganfall ist in Deutschland der häufigste Grund dauerhafter Behinderung und die fünfthäufigste Todesursache; ca. $20 \%$ aller über 65 -Jährigen sind davon betroffen (Statistisches Bundesamt 2006, Mosso und Baumgartner 2000).

Im Wesentlichen stehen zur Therapie der Karotisstenose drei Optionen zur Verfügung. Zum einen die medikamentöse Therapie, die auf die beeinflussbaren Faktoren in der Pathogenese der Gefäßwandschädigung abzielt und die Ausbildung von Thromben verhindert. Zum anderen zwei Therapien, die vordergründig die Stenose mechanisch behandeln (Bates et al. 2007). Die beiden Verfahren sind die Stentgeschützte Perkutane Angioplastie der Karotiden (SPAC) und die Karotisendarterektomie (TEA). Beide Verfahren konkurrieren miteinander in zahlreichen, zum größten Teil prospektiven (NASCET steering committee 1991, Barnett et al. 1998, Halliday et al. 2004, ECST group 1991, ECST group 1998, Wholey et al. 2003, Theiss et al. 2004), aber auch randomisierten Studien (Naylor et al. 1998, Alberts 2001, Brooks et al. 2001 und 2004). Die Erkenntnisse aus diesen Studien haben dazu beigetragen, beide Verfahren zu akzeptieren und die Stärken der einzelnen Verfahren für den Patienten optimal zu nutzen. Daher geht es nicht mehr um die Frage, welches der beiden Verfahren besser ist, sondern welches Verfahren bei welchem Patienten das beste Nutzen-Risiko-Verhältnis hat. Um die Risiken der Therapie vor Intervention beurteilen zu können, wird versucht, aus Klinik, demografischen Daten der Patienten und Bildgebung prognostische Parameter zu gewinnen. Die CT-Angiographie (CTA) ist in der Lage, die Stenose und ihre Plaques in sehr guter Qualität schnell und nichtinvasiv darzustellen, und stellt damit ein sehr wichtiges Hilfsmittel in der Beurteilung der Karotisstenose dar. Plaquezusammensetzung und Plaquemorphologie, insbesondere Ulzerationen der 
Plaques (Saba et al. 2007), stehen im Verdacht, einen Einfluss auf die Komplikationsrate bei Durchführung einer SPAC zu haben. Die Diffusionsgewichtete Magnetresonanztomographie (DWI-MRT) erlaubt, diese periinterventionellen Komplikationen, wie z.B. mikroembolische Infarkte, zu diagnostizieren und zu beurteilen (Barth et al. 2000). 


\section{Grundlagen}

\subsection{Schlaganfall und TIA}

\subsubsection{Epidemiologische Daten}

Der Schlaganfall zählt zu den häufigsten Erkrankungen in Deutschland und ist mit nahezu 29000 Todesfällen pro Jahr die fünfthäufigste Todesursache in der BRD überhaupt (Statistisches Bundesamt 2006). Die Inzidenz nimmt dabei mit steigendem Lebensalter zu. In Industrieländern liegt die Inzidenz des Schlaganfalls bei 300/100000/Jahr in der Altersgruppe von 55-64 Jahren und bei 800/100 000/Jahr in der Altersgruppe von 65-74 Jahren (Scheinert und Schmidt 2007). Männer sind in den Altersgruppen <85 Jahren etwa 30\% häufiger betroffen als Frauen (Mosso und Baumgartner 2000).

Für die Volkswirtschaft hat der Schlaganfall eine immense Bedeutung. Die direkten Kosten für Akutbehandlung, Rehabilitation und weitere Therapien stellen eine hohe Belastung für das Gesundheitssystem dar. Nicht zu vernachlässigen sind dabei die indirekten Kosten, die durch Ausfall der Produktivität der Betroffenen entstehen. In den USA wurden für das Jahr 2006 die indirekten und direkten Kosten des Schlaganfalls auf 57,9 Milliarden Dollar geschätzt (Thom et al. 2006).

$5-12 \%$ aller Schlaganfälle sind auf eine behandelbare signifikante Obstruktion im Bereich der Karotisstrombahn zurückzuführen (Struffert et al. 2004). Damit stellt die Karotisstenose eine wichtige Ursache von Schlaganfällen dar. Die Häufigkeit von $>50 \%$ igen Karotisstenosen liegt in der älteren Normalbevölkerung bei Frauen zwischen 5-7\% und bei Männern zwischen 6-11\% (Mosso und Baumgartner 2000). Ursache der Karotisstenose ist dabei hauptsächlich wie auch bei der koronaren Herzkrankheit (KHK) die Atherosklerose (Riede et al. 2004).

\subsubsection{Einteilung und Klinik}

Zerebrale Ischämien können nach verschiedenen Kriterien eingeteilt werden. Nach dem zeitlichen Verlauf unterscheidet man die transitorisch ischämische Attacke (TIA) mit einer Dauer unter $24 \mathrm{~h}$ vom Schlaganfall mit einer Dauer über $24 \mathrm{~h}$ (Scheinert und Schmidt 2007). 
Trotz ihrer kurzdauernden Symptomatik sind TIA ernst zu nehmende medizinische Notfälle. $11 \%$ dieser Patienten bekommen einen Schlaganfall innerhalb von 90 Tagen nach einer TIA; davon die Hälfte während der ersten 2 Tage (Johnston et al. 2000). Der Schlaganfall lässt sich weiter unterteilen in minor und major stroke. Ein minor stroke stellt ein neurologisches Defizit dar (okulär oder zerebral), das länger als $24 \mathrm{~h}$ persistiert und das sich entweder nach 30 Tagen wieder zurückbildet oder zur NIH stroke scale nicht mehr als 3 Punkte beiträgt. Ein major stroke ist dementsprechend ein Defizit, das länger als 30 Tage persistiert oder mehr als 3 Punkte zur NIH stroke scale beiträgt (Gröschel et al. 2008).

Eine andere Klassifikation bezieht sich auf die Infarktmorphologie. Territorialinfarkte sind durch embolische oder lokale thrombotische Verschlüsse ausgelöste Ischämien, die sich keilförmig auf das Versorgungsterritorium des betroffenen Gefäßes beschränken. Je nach Höhe des Verschlusses kann weiter in totalen und subtotalen Territorialinfarkt unterteilt werden. Zentrale Territorialinfarkte entstehen bei partieller Kollateralisierung des Randbezirks. Hämodynamische Infarkte entstehen aufgrund einer hämodynamisch relevanten Stenose der zuführenden Gefäße. Unterschieden werden kann dabei in Endstrominfarkten von distalen penetrierenden Arterien und Grenzzoneninfarkten, welche zwischen Versorgungsgebieten zweier oder mehrerer großer Gefäße liegen (Reiser et al. 2004).

Die Klinik richtet sich nach dem Versorgungsgebiet der betroffenen Arterie. Während Verschlüsse im Bereich des vertebrobasilären Versorgungsgebietes meist mit Vigilanzstörungen einhergehen, bleibt bei Verschlüssen der Karotiden das Bewusstsein erhalten. Häufigste Symptome sind Sehstörungen wie Amaurosis fugax, kontralaterale Arm- oder Beinschwäche und Sprachstörungen (Bates et al. 2007).

\subsection{Karotisstenose}

\subsection{1. Ätiologie und Pathogenese}

Die Ätiologie von Schlaganfällen ist vielfältig und trotzdem kann häufig schon anhand der Infarktmorphologie auf die zugrunde liegende Ursache geschlossen werden.

Die häufigste Ursache für einen Schlaganfall ist mit etwa 30\% die Embolie, entweder aus den vorgeschalteten Arterien oder aus dem Herzen (Poeck und Hacke 2001). Ein Sonderfall ist die paradoxe Embolie, bei der der Thrombus aus dem venösen 
Stromgebiet kommt und nur über einen Shunt in das arterielle System gelangt. Die Atherosklerose hat in der Ätiologie des Schlaganfalls eine besondere Bedeutung, da sie in wechselseitiger Beziehung zu vielen verschiedenen Ursachen des Schlaganfalls steht. Sie ist beteiligt an der Entstehung von Embolien aus dem arteriellen Stromgebiet, lokalen arteriellen Thrombosen und an arteriellen Gefäßwanddissektionen. Für unsere Studie ist ihr Einfluss an der Entstehung von Obstruktionen der Karotiden entscheidend, die für 5-12\% aller Schlaganfälle als Ursache stehen (Scheinert und Schmidt 2007). Dabei kann es auf zwei Wegen zu einem ischämischen Ereignis kommen. Zum einen führt die Obstruktion zu einer Minderperfusion des Versorgungsareals des betroffenen Gefäßes und zum anderen, viel häufiger, können sich Thromben an der Plaque bilden, die zu einer Embolie eines Endstromgebietes führen.

Karotisstenosen sind meist asymptomatisch. Ein Strömungsgeräusch über den Karotiden kann bei $4-5 \%$ der Patienten zwischen 45 und 80 Jahren festgestellt werden (Bates et al. 2007). Karotisstenosen von mindestens 50\% können sogar bei $7 \%$ der Männer und 5\% der Frauen über 65 Jahren diagnostiziert werden (O'Leary et al. 1992). Das Risiko des Fortschreitens der Stenose liegt bei 9,3\% pro Jahr (Muluk et al. 1999). Risikofaktoren hierfür sind ipsilaterale oder kontralaterale Stenosen der A. carotis interna $(\mathrm{ACl})$ über $50 \%$, ipsilaterale Stenosen der A. carotis externa (ACE) über $50 \%$ und arterieller Hypertonus über $160 \mathrm{mmHg}$ systolisch. Dabei hängt das Risiko, einen Schlaganfall zu erleiden, nicht nur vom Grad der Obstruktion ab, der bei Zunahme um 10\% das Schlaganfallrisiko etwa um den Faktor 1,3 erhöht, sondern vor allem auch vom Ausmaß der Kollateralisation, dem Vorliegen kardiovaskulärer Risikofaktoren, dem Vorhandensein subklinischer zerebraler Ischämien, der Plaquemorphologie und vielen anderen klinischen Merkmalen. Das Risiko, einen Schlaganfall zu erleiden, liegt bei einer asymptomatischen Stenose bei 1-2\%/Jahr. Bei einer symptomatischen Stenose steigt es auf 26\%/2Jahre und am höchsten ist es unmittelbar nach einem initialen ischämischen Ereignis (Scheinert und Schmidt 2007, Barnett et al. 1998). Paradoxerweise haben Patienten mit einer subtotalen Okklusion ein niedrigeres Schlaganfallrisiko, das zwischen 8\% für 5 Jahre (Rothwell et al. 2003) und 11\% für ein Jahr (Morgenstern et al. 1997) liegt. 


\subsubsection{Einflussfaktoren auf die Plaquemorphologie}

Plaques in Karotisstenosen gelten als Folge von Atherosklerose. Atherosklerose wiederum ist eine systemische Krankheit der Arterien, die vor allem die Intima mittlerer bis großer Arterien verändert, wie z.B. Karotiden, Aorta, Herzkranzgefäße und periphere Arterien (Fuster et al. 2005a). Die Plaques bestehen hauptsächlich aus Kollagen, Proteoglykanen, Fibronektin, kristallinisiertem Cholesterol, Cholesterinester, Phospholipiden und Zellmaterial wie Makrophagen, T-Lymphozyten und glatten Muskelzellen (Daugherty und Rateri 2002). Hierum bildet sich thrombotisches Material wie Thrombozyten- und Fibrinablagerungen (Stary et al. 1995). Die Plaquemorphologie und -stabilität wird dabei wesentlich vom Verhältnis der Bestandteile zueinander beeinflusst (Fuster et al. 2005a).

Am Beginn der Läsion steht die endotheliale Dysfunktion durch Scherkräfte an der Gefäßwand, ausgelöst wiederum durch turbulenten Blutfluss; vorzugsweise an Biegungen und Bifurkationen (Fuster et al. 2005a, Ravensbergen et al. 1998). Arterielle Hypertonie fördert dabei die mechanischen Scherkräfte. Einen erheblichen Einfluss auf die Plaquemorphologie hat der Fettstoffwechsel. Lipide, insbesondere Cholesterin, gelangen über low-density-Lipoproteine (LDL) in die Intima, wo sie oxidiert werden und sich ablagern (Steinberg et al. 1989). Eine Interaktion mit Proteoglykanen löst eine chemische Modifikation und lokale Entzündung aus (Libby 2001). Das high-density-Lipoprotein (HDL) hingegen fördert den Abtransport von Cholesterin (Khalil et al. 2004).

Die Stadien der atherosklerotischen Läsionen können nach der American Heart Association (AHA) klassifiziert werden (s. Abbildung 01). Da die Klassifikation klinische Gesichtspunkte nicht berücksichtigt, wurde mehrfach versucht, sie zu überarbeiten und zu optimieren. Stary et al. (1995) modifizierten die AHAKlassifikation nach klinisch relevanten Stadien. Sinnvoller erscheint allerdings eine Einteilung der Plaquevulnerabilität nach high-risk und low-risk (Schaar et al. 2004).

Die Einteilung nach Plaquevulnerabilität hat eine besondere klinische Bedeutung, da die Ruptur der vulnerablen Plaque zu einer Freisetzung von thrombogenem Material und damit zu einer akzelerierten Größenzunahme der Plaque führt (Davies und Thomas 1985, Ding et al. 2008). Dies wiederum verursacht eine lokale Thrombose und erhöht das Risiko von arteriellen Embolien (Fuster et al. 2005a, Virmani et al. 
2002). Beides resultiert im Falle der Karotisstenose in einer zerebralen Minderperfusion bzw. in einem Schlaganfall.

\section{Different Types of Vulnerable Plaque}

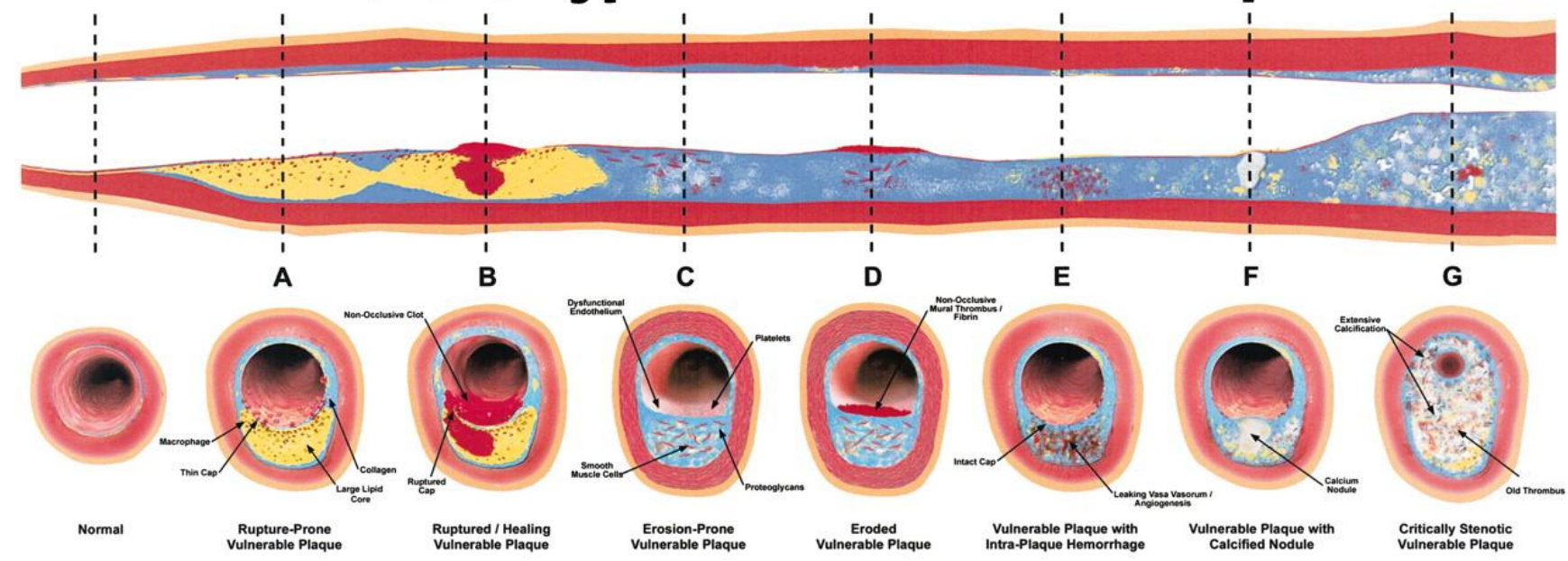

Abbildung 01: Die Klassifikation der American Heart Association (AHA) teilt verschiedene Typen von vulnerablen Plaques als Ursache des akuten Koronarsyndroms ein. A: Rupturgefährdete Plaque mit großem Lipidkern und dünner fibröser Kappe, die von Makrophagen infilftriert wird. B: Rupturierte Plaque mit subokklusivem und frühorganisiertem Thrombus. C: Zur Erosion neigende Plaque mit Proteogykanmatrix und Infiltration von glatten Musklezellen. D: Erodierte Plaque mit subokklusivem Thrombus. E: Hämorrhagie der Plaque aus vasa vasorum. F: Kalzifierter Herd, der in das Gefäßlumen hineinragt. G: Chronisch stenosierende Plaque, gezeichnet durch starke Kalzifikation, alte Thrombosierung und exzentrisches Lumen (Naghavi et al. 2004, S.1669).

\subsubsection{Bildgebende Diagnostik}

\subsubsection{Sonographie}

Die Duplexsonographie steht bei der apparativen Diagnostik beim Verdacht auf Stenosierungen der supraaortalen Strombahn an erster Stelle. Praktikabilität, Nichtinvasivität und geringe Kosten sind der entscheidende Vorteil gegenüber anderen Verfahren. Es gibt drei Sonographieverfahren, die sich in der Praxis bewährt haben: die farbkodierte Duplexsonographie, die continuous-wave-(Cw-)Sonographie und der pulsed-wave-Doppler der Duplexsonographie. Stenosegrad und Plaquemorphologie können durch Bestimmung der Flussgeschwindigkeit aus dem Doppler (Aliasing-Phänomen) und durch morphologische Informationen aus dem BBild gut beurteilt werden. Durch einen zusätzlichen transkraniellen Ultraschall kann auch die hämodynamische Wirksamkeit der Stenose untersucht werden. Die Sensitivität der Sonographie liegt hier bei 80\%, bei einer Spezifität von 82\% (Ranke et al. 1999). Damit hat sich die Utraschalldiagnostik der Halsgefäße als 
Screeningverfahren durchgesetzt. Trotzdem gibt es außer der Erfahrung des Untersuchers eine Reihe von patientenspezifischen Faktoren, die die Qualität der Untersuchung beeinträchtigen, z.B. Herzfunktion, Blutdruck, Vorliegen eines kontralateralen $\mathrm{ACl}$-Verschlusses, unterschiedliche Karotisdurchmesser und viele mehr. Insgesamt ermöglicht die Sonographie eine gute Verlaufskontrolle und die Abschätzung über die Dringlichkeit von Interventionen und über das Vorhandensein von atherosklerotischen Plaques, die Ursprung einer Embolie sein können.

\subsubsection{CT-Angiographie}

Die Möglichkeiten des Einsatzes der Computertomographie in der Gefäßdarstellung haben sich seit den 90er Jahren stark gewandelt. Durch die Einführung der mehrzeiligen Computertomographen (multislice-CT) ist eine deutliche Erhöhung der Zeit- und Ortsauflösung zu vermerken.

Bei der CT-Angiographie (CTA) wird Kontrastmittel gleichmäßig gesteuert intravenös appliziert. Das Kontrastmittel gelangt durch das Herz in den arteriellen Stromkreis und damit in die Karotiden und zerebralen Arterien. So lässt sich das gesamte supraaortale Gefäßsystem darstellen, was gerade bei Tandemstenosen, also hintereinander geschalteten Stenosen, ein Vorteil ist. Vergleiche mit der Digitalen Subtraktionsangiographie (DSA) zeigen, dass die CTA die Lage der höchstgradigen Stenosen ebenso gut beschreiben kann (Chen et al. 2004). In Notfallsituationen bietet sie den Vorteil, dass bei dieser Untersuchung auch intrazerebrale Blutungen ausgeschlossen werden können. Ein weiterer wichtiger Aspekt ist die minimale Invasivität dieses Verfahrens mit einer Risikoreduktion gegenüber invasiven Verfahren. Je nach Zeiligkeit eines CT-Scanners kann die Untersuchungszeit bei 415 s liegen.

Ebenso besteht, bezogen auf prädiktive Parameter der Stenose, eine gute Vergleichbarkeit mit anderen Verfahren. Vergleiche zwischen DSA und CTA haben gezeigt, dass der Stenosegrad gemessen mit der CTA eine hohe Korrelation mit der DSA hat (Silvennoinen et al. 2007). Vorteile bietet die CTA in der Beurteilung der Plaqueoberfläche. Durch axiale Projektionen und verschiedene Rekonstruktionen wie maximum intensity projection (MIP), multiplanar reconstruction (MPR), shaded surface display (SSD) und volume rendering (VR) konnte eine starke Korrelation in 
der Beurteilung von Plaqueulzerationen im Vergleich zum chirurgischen Präparat nach TEA festgestellt werden (Saba et al. 2007).

\subsubsection{MR-Angiographie}

Wie keine andere Technik hat die MR-Angiographie (MRA) von den technischen Erneuerungen in der Bildgebung der letzten Jahre profitiert. Durch die MRA ist es wie durch die CTA möglich, intrathorakale und intrakranielle Gefäße darzustellen, die durch die Dopplersonographie nicht erreicht werden können, jedoch wird ihre Bildqualität stark durch Atmungs- und Bewegungsartefakte beeinträchtigt (Wutke et al. 2002). Lange Untersuchungszeiten schwächen die Bedeutung der MRA; jedoch haben neue Algorithmen der Bildrekonstruktion und höhere Feldstärken der Geräte zur Verkürzung dieser Zeit und zur Erhöhung der Bildqualität beigetragen (AlvarezLinera et al. 2003, Wutke et al. 2002). Die Bilder lassen sich wie auch die der CTA dreidimensional, axial, sagittal und koronar befunden. Im Gegensatz zur CTA ist die MRA nicht mit ionisierenden Strahlen oder nephrotoxischen Kontrastmitteln vergesellschaftet. Trotzdem gibt es zahlreiche Kontraindikationen, wie z.B. Klaustrophobie, Schrittmacher und Defibrillatoren, Metallimplantate und in gewissem Maße auch Adipositas (Bates et al. 2007). Des Weiteren ist diese Untersuchung trotz ihrer hohen Aussagekraft in der routinemäßigen Anwendung nicht kosteneffizient (Buskens et al. 2004).

Interessant ist die Darstellung der Plaquemorphologie mittels MRT unter Zuhilfenahme spezieller Oberflächenspulen und die dadurch mögliche Plaquebildgebung mit Bestimmung von Bindegewebs- und Fettanteilen, sowie Ulzerationen und Einblutungen der Plaques (Glor et al. 2003). Dies ist gegenwärtig Fokus zahlreicher Studien, hat jedoch noch keinen Einzug in die Routinediagnostik gefunden.

\subsubsection{Digitale Subtraktionsangiographie}

Die Digitale Subtraktionsangiographie (DSA) über einen intraarteriellen Katheter ist der Goldstandard in der Beurteilung von Erkrankungen der Karotiden (Scheinert und Schmidt 2007). Durch sie erhält man wichtige Informationen über die Anatomie des Aortenbogens, die Konfiguration der Gefäßabgänge und über den Gefäßverlauf 
sowie über atherosklerotische Schäden der Gefäße. In der Diagnostik der Karotisstenose kommt ihr eine wichtige Bedeutung bei der Bestimmung des Stenosegrades zu, die nach 3 Messmethoden, NASCET, ECST und CC, durchgeführt wird. Diese unterscheiden sich hinsichtlich des Referenzdurchmessers der Stenose (s. Abbildung 04), weswegen die Stenosegrade voneinander abweichen können. Durch Darstellung der Flussverhältnisse ermöglicht die DSA eine Beurteilung der Funktionalität einer Stenose. Die Indikation für eine interventionelle oder operative Versorgung wird mit der DSA gestellt, wobei per Konvention die Messung nach NASCET berücksichtigt wird (Bates et al. 2007). Bei entsprechender Vorbereitung kann sich dieser Untersuchung eine SPAC anschließen.

Als invasives Verfahren birgt die Katherangiographie allerdings auch Risiken. Neben den allgemeinen Komplikationen durch den Zugangsweg, wie Leistenhämatom, Infektion, Kontrastmittelnephropathie und anaphylaktischen Reaktionen durch eine Kontrastmittelallergie, sind vor allem arterielle Thrombembolien zu nennen. Das Risiko für Patienten mit symptomatischer Karotisstenose, bei der diagnostischen Angiographie einen Schlaganfall zu erleiden, liegt zwischen 0,5 und 5,7\%; für eine TIA sogar zwischen 0,6 und 6,8\% (Connors et al. 2005). Jüngere Studien berichten ein niedrigeres Risiko (Fayed et al. 2002, Leonardi et al. 2005), was womöglich mit verbesserter Technik und Material und dem Einsatz von Heparin und Thrombozytenaggregationshemmern zusammenhängt (Bates et al. 2007).

\subsubsection{Therapie}

\subsubsection{Karotisendarterektomie}

1954 wurde von Eastcott erstmals ein Fall einer Arterektomie bei hochgradiger Karotisstenose mit End-zu-End-Anastomose publiziert und weitere folgten. Die damit behandelten Patienten erlitten nach der Intervention keine TIA mehr. Seit den Studien der North American Symptomatic Carotid Endarterectomy Trial (NASCET) und der darauffolgenden Europeen Carotid Surgery Trial (ECST) hat sich die Endarterektomie in der Behandlung von symptomatischen Karotisstenosen gegenüber der rein Medikamentösen mit ASS und Clopidogrel durchgesetzt (NASCET steering committee 1991, ECST group 1991 und 1998). Als Indikation für eine TEA empfiehlt die American Heart Association (AHA) einen Stenosegrad von 
50-99\% für symptomatische Patienten, bei denen das Risiko eines perioperativen Schlaganfalls oder Todes unter 6\% liegt, und einen Stenosegrad von $60-99 \%$ bei asymptomatischen Patienten, bei denen das Risiko von Schlaganfall oder Tod unter $3 \%$ liegt. Hiervon abweichend wird die Indikation für asymptomatische Patienten in einigen Zentren strenger gestellt. Diese Empfehlungen ergeben sich aus der 5Jahres-Risikoreduktion von Schlaganfällen bei symptomatischen 70 bis $99 \%$ igen Stenosen um $48 \%$ bzw. bei 50 bis $69 \%$ igen symptomatischen Stenosen um $28 \%$ gegenüber den perioperativen Komplikationen wie z.B. Herzinfarkt (NASCET group 1991, ECST group 1991, Massachusetts Medical Society 1991). Bei asymptomatischen Stenosen $>60 \%$ konnte das Risiko nur von $12 \%$ auf $3 \%$ gesenkt werden (Hallyday et al. 2004, Struffert et al. 2004).

Es stehen mit der Längsarterotomie ggf. mit Patchplastik (TEA) und der Eversionsendarteriektomie (EEA) zwei Verfahren zur Verfügung (Rantner und Fraedrich 2005). Die Wahl richtet sich nach Ausdehnung und Plaquemorphologie. Dabei werden die Aa. carotis communis, interna und externa freigelegt und nach Heparingabe abgeklemmt. Bei der TEA werden die Aa. carotis communis und interna längs eröffnet und die Plaque in der Ebene der Membrana elastica externa desobliteriert. Die Arterien werden dann entweder durch Direktnaht oder durch Patchplastik wieder verschlossen. Bei der EEA werden die Arterien bis auf Ebene der Adventitia evertiert. Wichtige Risiken dieses Eingriffs sind Schlaganfall durch Mikroembolien oder hämodynamisch verursacht und Läsionen der Hirnnerven, N. hypoglossus und N. laryngeus recurrens, die zu Dysästhesien oder Heiserkeit führen, aber meist reversibel sind.

\subsubsection{Stentgeschützte Perkutane Angioplastie der Karotiden}

Die Stentgeschützte Perkutane Angioplastie der Karotiden (SPAC), auch Carotisangioplastie (CAS) oder stent-protected carotid angioplasty (SPCA), ist ein alternatives Verfahren zur TEA, vor allem bei Patienten, bei denen eine TEA ein hohes Komplikationsrisiko darstellt. Der therapeutische Erfolg beruht auf zwei Punkten. Zum einen soll durch den Stent und Ballondilatation das stenosierte Gefäß wieder erweitert werden und somit die Hämodynamik gebessert werden. Zum anderen wird die Plaque, thrombotisches Material und die geschädigte Gefäßwand hinter dem Stent fixiert und soll somit Thrombembolien verhindern. Die Vorteile 
liegen vor allem in der geringen Invasivität und damit Vermeidung von Komplikationen. Eine Vollnarkose ist in der Regel bei diesem Verfahren nicht notwendig. Eine geringere Komplikationsrate als bei der TEA ist allerdings nicht unumstritten. Ohne Alternative steht sie in der Behandlung von sub- bzw. intrapetrösen und intrakraniellen Stenosen zur Verfügung. Ein weiterer Vorteil ist ihre diagnostische Bedeutung vor und viel wichtiger nach der Stentapplikation, da die zerebrale Perfusion beurteilt werden kann.

Mit einem Kathetersystem kann die Karotis über einen Zugang in der Leiste erreicht werden und ein Stent in die Stenose appliziert werden. Vor Stentapplikation oder obligatorisch danach wird die Stenose per Ballondilatation erweitert (zur genauen Durchführung der SPAC sei auf den Methodenteil verwiesen).

Komplikationen dieser Behandlung ergeben sich vor allem daraus, dass die Plaque anders als bei der TEA nicht entfernt wird, sondern durch den Stent quasi nur fixiert wird. Mikrothromben, bzw. kleine Plaqueteile können beim Passieren der Stenose mit dem Führungsdraht, bei Applikation des Stents oder bei der Nachdilatation abgeschwemmt werden (Ohki et al. 2001). Durch Verschlüsse der Endarterien können sie Mikroinfarkte verursachen, die üblicherweise keine Symptomatik hervorrufen. Des Weiteren besteht die Gefahr von Luftembolien.

\subsubsection{Zerebrale Protektionssysteme}

Protektionssysteme, die bei einer SPAC das Auftreten von Mikroembolien verhindern sollen, sind seit 1987 in der Entwicklung und seit 1996 im klinischen Einsatz (Théron et al. 1990). In ihrer Weiterentwicklung sind sie anerkannte und für einige Interventionalisten fast unabdingbare Verfahren. Verschiedene Systeme mit sehr guten Ergebnissen sind in der klinischen Anwendung, doch sie sind auch nicht komplikationslos. Einen vollständigen Schutz vor Mikroembolien bieten sie nicht. So scheint es sehr sinnvoll, Indikationen für den Einsatz von Protektionssystemen genauer zu erforschen, um so die Komplikationsrate der SPAC weiter zu senken.

Es gibt im Wesentlichen zwei verschiedene Arten von Systemen zur Protektion vor Mikroembolien (Müller-Hülsbeck 2002). Die passagere Ballonokklusion, wie z.B. bei Percusurge Guardwire ${ }^{\mathrm{TM}}$ (Sunnyvale, CA, USA), ist eine Technik, bei der über einen Führungsdraht, der die Stenose passiert, ein Ballon distal der Stenose platziert wird. Eine Inflation des Ballons führt zu einer temporären Okklusion, die ein Verschleppen 
von thrombotischem Material während der Stenteinlage und der Nachdilatation verhindert. Durch einen zusätzlichen Aspirationskatheter kann das Material gespült, aspiriert und damit aus dem Gefäß entfernt werden. Nach Stentapplikation kann der Ballon deflatiert und wieder eingezogen werden.

Die passagere Filtereinlage, wie z.B. Angioguart ${ }^{\mathrm{TM}}$ (Cordis, Roden, Niederlande), Filter Wire EX ${ }^{\mathrm{TM}}$ (Boston Scientific, Natick, MA, USA), NeuroShield ${ }^{\mathrm{TM}}$ (MedNova, Horsham, UK) und Accunet ${ }^{\mathrm{TM}}$ (Guidant, Santa Clara, CA, USA) funktionieren auf ähnliche Weise wie die Ballonokklusion. Auch sie werden durch den Führungsdraht an der Stenose vorbei distal platziert. Sie können regenschirmartig ein- oder ausgeklappt werden. Sie okkludieren allerdings das Gefäß nicht komplett wie die Ballonokklusionssysteme, da ihre Membran wie ein Filter wirkt. Trotzdem werden sie nur bei Bedarf ausgeklappt. Nach Stentapplikation werden sie wieder eingeklappt und können mit dem gefangenen thrombotischen Material aus dem Gefäß entfernt werden. In der Abteilung für Neuroradiologie des Uniklinikums Göttingen wird einzig der Filter Wire EX'⿳⺈ v verwendet.

Die genannten Verfahren weisen einige Einschränkungen auf. So ist die Ballonokklusion nicht bei ipsilateralem A.-cerebri-ant.-Verschluss oder kontralateralem ACl-Verschluss geeignet. Bei fehlender Kollateralisation kann es bei der Unterbrechung des Blutflusses zu einer zerebralen Minderperfusion kommen. Des Weiteren kann es beim Spülen des thrombotischen Materials zu einem Reflux in die ACE kommen. Je nach Kollateralisation zu anderen Gefäßen kann es andernorts zum thrombotischen Verschluss kommen (Théron et al. 1990). Wesentlich häufiger sind Komplikationen durch Zurückbleiben von thrombotischem Material nach Aspiration, wenn es sich zwischen Ballon und Gefäßwand fixiert. 


\section{Fragestellung}

Die SPAC ist ein alternatives Verfahren zur TEA bei symptomatischen und asymptomatischen Karotisstenosen. Derzeit ist noch nicht abschließend geklärt, welches der Verfahren dem anderen überlegen ist. Unumstritten ist die Überlegenheit der SPAC bei intrakraniellen Stenosen und bei Patienten, bei denen eine Operation und die damit verbundene Vollnarkose ein hohes Risiko darstellen. Durch den Einsatz von Protektionssystemen konnte das Auftreten periinterventioneller Mikroembolien weiter gesenkt werden, doch auch diese bringen neue Komplikationen mit sich. Es wäre daher wünschenswert und wichtig, prognostische Faktoren aus der Klinik der Patienten und aus der Bildgebung zu erhalten, um Kriterien zu entwickeln, nach welchen der Patient eher dem einen oder dem anderen Verfahren zugeführt werden kann, bzw. um eine individuelle Risikoabschätzung für die Intervention geben zu können.

Es gibt Hinweise darauf, dass die Plaquezusammensetzung der Karotisstenose einen wesentlichen Einfluss auf das Auftreten von mikroembolischen Ereignissen während, bzw. nach SPAC hat. Wir vermuten, dass ein hoher Weichplaqueanteil das Risiko für Mikroembolien erhöht. Durch eine Bestimmung des Weichplaqueanteils in der CT-Angiographie und der Läsionslast in der DWI-MRT nach SPAC wollen wir diesen Zusammenhang untersuchen.

Des Weiteren ist bekannt, dass die Morphologie der Plaque ebenfalls einen Einfluss auf das Auftreten von mikroembolischen Ereignissen nach SPAC hat. Ulzerationen und Einblutungen innerhalb der Plaque scheinen mit einem erhöhten Risiko für Mikroembolien einherzugehen. Durch die Klassifikation der Plaquemorphologie nach Lovett et al. (2004), die auf Ulzerationen der Plaque fokussiert, wollen wir diesen Zusammenhang untersuchen. Da diese Klassifikation von Lovett et al. ursprünglich für die DSA entwickelt wurde, wollen wir zeigen, dass sie sich ebenso in der CTA reproduzieren lässt. Dafür wollen wir die Ergebnisse der Klassifikation in beiden Verfahren miteinander vergleichen. In Subgruppenanalysen wollen wir untersuchen, ob die Plaquemorphologie nach dieser Klassifikation ebenso Hinweise auf ein erhöhtes Risiko von Mikroembolien geben kann.

Einen weiteren wichtigen Zusammenhang, den wir untersuchen wollen, sind technische Details bei der Durchführung der SPAC. Insbesondere zu nennen sind Länge der Intervention, Anzahl der Kontrastmittelserien, Entlüftung des Stentsystems 
und der Einsatz von zerebralen Protektionssystemen. Außer diesen sind noch weitere Risikofaktoren für Mikroembolien nach SPAC bekannt. Daher wollen wir sowohl klinische und demografische Daten der Patienten, als auch Daten aus CTA und DSA auf einen Zusammenhang mit Läsionen in der DWI-MRT untersuchen.

Ziel unserer Studie ist es, prädiktive Faktoren für mikroembolische Ereignisse während und nach der Durchführung einer SPAC zu identifizieren und ihren Einfluss auf die Komplikationsrate dieser Intervention zu untersuchen. 


\section{Methode}

\subsection{Studiendesign und Patientenkollektiv}

In unsere prospektive Studie wurden 46 Patienten mit einer symptomatischen oder asymptomatischen extrakraniellen Stenose der A. carotis interna ( $\mathrm{ACl}$ ) und/oder $\mathrm{A}$. carotis communis (ACC) im Zeitraum von Juli 2006 bis März 2008 am Universitätsklinikum Göttingen eingeschlossen. 2 der 46 Patienten wurden beidseits an den Karotiden behandelt. 36 Patienten waren männlich, 10 weiblich. Das Alter der Patienten lag zwischen 49 und 84 Jahren mit einem Mittelwert von 67,85 Jahren. Alle Patienten wurden erfolgreich mittels einer SPAC behandelt. Vor der SPAC wurde eine CTA der hirnzuführenden Gefäße mit Kontrastmittel durchgeführt. Innerhalb von 48h jeweils vor und nach SPAC wurde ein kranielles MRT mit Diffusionsgewichtung angefertigt.

Ausgeschlossen waren Patienten, die ipsilateral der Stenose durch TEA oder SPAC vorbehandelt waren oder Verwachsungen der Karotiden anderer Genese hatten, z.B. bestrahlungsassoziierte Stenosen der Karotiden. Ebenso ausgeschlossen waren Patienten, die aufgrund von Kontraindikationen keiner MRT- oder CTA-Untersuchung zugeführt werden konnten.

Die initiale Diagnose einer Karotisstenose wurde von der Abteilung für Neurologie des Klinikums Göttingen anhand von klinischen Gesichtspunkten und Stenosegraden aus der Dopplersonographie gestellt. Nach weiterer Diagnostik durch CTA und eventuell DSA wurden die Patienten unter der Berücksichtigung klinischer Entscheidungskriterien und im Konsens mit den behandelnden Neurologen einer SPAC zugeführt.

Alle Patienten haben schriftlich in die Teilnahme an der Studie eingewilligt und sind über alternative Verfahren wie TEA oder konservative medikamentöse Therapie aufgeklärt worden. Unsere Studie wurde bei der Ethikkommission der Universitätsmedizin Göttingen beantragt und von ihr genehmigt. 


\subsection{SPAC}

Die Patienten wurden vor der Intervention ausführlich über Art, Umfang, Risiken und Alternativen des Eingriffs aufgeklärt und willigten in Selbigen ein. Die Intervention erfolgte in anästhesiologischem Standby und bei Bedarf in leichter Sedierung durch Prämedikation mit einem Benzodiazepin. Die SPAC wurden von einem Team aus erfahrenen interventionellen Neuroradiologen durchgeführt. Bei allen Interventionen erfolgte der Zugang über den perkutanen femoralen Weg. Es wurde eine 7-FSchleuse eingelegt und ein 7-F-Mach1-Katheter eingeführt. Nach selektiver Sondierung $\operatorname{der}$ A. carotis communis wurden mittels Kontrastmittelapplikation Standardserien der Karotisbifurkation, der Stenose sowie der intrakraniellen Zirkulation angefertigt. Vor Einführen des Stents wurde Heparin (100 U/kgKG) intravenös appliziert; je nach ACT-Zeit wurde die Dosierung angepasst. Nach Sondierung der Stenose mit einem Mikrodraht wurde der Stent platziert.

Dabei wurden folgende Stents verwendet:

- Boston Scientific Carotid Wallstent ${ }^{\mathrm{TM}}$ (Boston Scientific, Natick, MA, USA)

- Sinus-Carotid-Conical-RX Stent ${ }^{\mathrm{TM}}$ (Optimed Global Care, Ettlingen, Deutschland)

- Cordis PRECISE RX Nitinol Stent System ${ }^{\mathrm{TM}}$ (Cordis Corporation, Miami, FL, USA)

Durch Nachdilatieren mit einem Sterling-Ballon wurde das Gefäßlumen im Bereich der Stenose rekonstruiert. Die Nachdilatation geschah unter medikamentöser Prophylaxe mit Atropin. In 17 von 48 Fällen wurde das Verfahren durch Einsetzen eines Protektionssystems erweitert. Dazu wurde die Stenose zuvor mit einem Boston Filter Wire EZ Embolic Protection System ${ }^{\mathrm{TM}}$ (Boston Scientific, Natick, MA, USA) vorsichtig passiert und das System im distalen zervikalen Segment der Arterie freigesetzt. Durch Anfertigen von diagnostischen Serien mit Kontrastmittel wurden die Lage des Stents und die intrazerebrale Perfusion vor, während und nach der Intervention beobachtet.

\subsubsection{Entlüftung und Durchspülung des Stentsystems}

Vor Applikation des Stents wurde das Kathetersystem gemäß der Empfehlung des Herstellers durchgespült (s. Abbildung 02). Dazu wurde eine 5-ml- Spritze, gefüllt mit 
steriler heparinisierter Kochsalzlösung, am T-Verbindungsstück (5) angebracht und die Kochsalzlösung kräftig in den kreisförmigen Raum zwischen dem koaxialen Innenkatheter und der äußeren Schleuse injiziert bis sie aus der äußeren Führungsdrahtöffnung (4) austrat. Dann wurde das Instrument mit den Fingern an der äußeren Führungsdrahtöffnung abgeklemmt und es wurde weitergespült bis die Kochsalzlösung aus der Katheterspitze und der äußeren Schleuse (1) austrat.

Zusätzlich zur Durchspülung des Katheters wurde in 8 Fällen der Boston Scientific Carotid Wallstent $^{\mathrm{TM}}$ auf eine in unserer Abteilung entwickelten Weise entlüftet. Dazu wurde der Stent in heparinisierter Kochsalzlösung 4 bis $5 \mathrm{Mal}$ zu 70\% freigesetzt und wieder eingezogen. Beide Maßnahmen, Durchspülung und Entlüftung, dienten der Prävention von sogenannten Luftembolien.

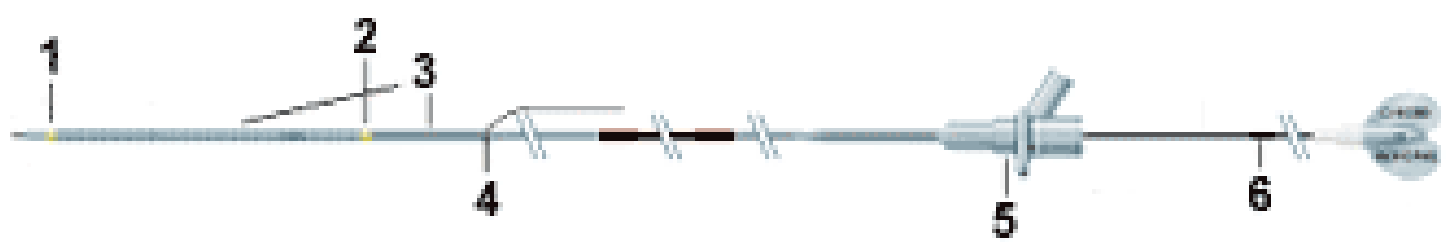

Abbildung 02: Boston Scientific Carotid Wallstent ${ }^{\mathrm{TM}}$. 1: Äußere Schleuse mit distalem Röntgenmarker des Innenkatheters. 2: Proximaler Röntgenmarker des Innenkatheters. 3: Außenkatheter. 4: Innere und äußere Führungsdrahtöffnung. 5: T-Verbindungsstück. 6: Platzierungsmarker.

\subsection{Plaquecharakterisierung in der CTA}

\subsubsection{Prinzip der CTA}

Die Computertomographie ist ein röntgenologisches Verfahren zur Erstellung von Transversaltomogrammen. Röntgenstrahlen, die auf das Untersuchungsobjekt appliziert werden, werden dabei von mehreren Detektoren erfasst (Laubenberger TH und Laubenberger J 1999). Die Differenz zwischen gesendeter und gemessener Intensität bestimmt den Schwächungskoeffizienten. Jeder Schwächungskoeffizient wird dabei nach Hounsfield einer bestimmten Hounsfield Einheit (HE) zugeordnet. Wasser gilt als Referenzwert mit einer HE von 0, Luft -1000 und Knochenkompakta $>250$ bis 3000 . 


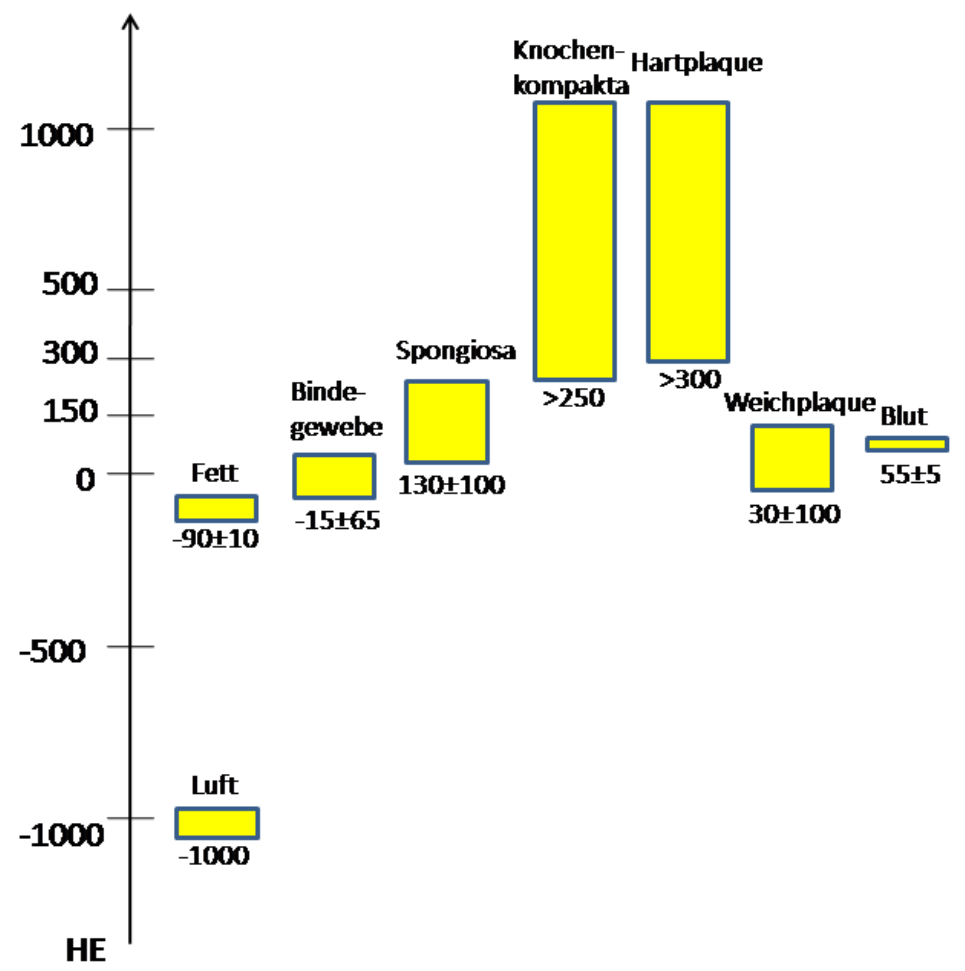

Abbildung 03: Dargestellt sind die Hounsfieldeinheiten (HE) verschiedener Gewebe. Von besonderem Interesse sind Hartplaque $>300 \mathrm{HE}$ und Weichplaque $30 \pm 100 \mathrm{HE}$.

Jedem Bildpunkt kann so ein Schwächungskoeffizient zugeordnet werden. Zur Bildgebung werden diese von dunkel nach hell in Grauwerte umgewandelt, also von hypodens nach hyperdens. Da der Untersucher maximal 20 Graustufen voneinander differenzieren kann, erfolgt die Darstellung der Schwächungswerte mit einer 10 bis 20-stufigen Graduierung. Diese Technik wird auch als Fensterung bezeichnet. Dabei wird eine Fensterlage festgelegt, also die HE, bei der die Mitte der Graustufen liegt und eine Fensterbreite, also die HE, die die Extreme der Graustufen bildet. Blut hat eine HE von $55 \pm 5$ und lässt sich dabei nur schwer von anderen Geweben wie Bindegewebe mit einer $\mathrm{HE}$ von $-15 \pm 65$ oder Muskelgewebe (40-60 HE) unterscheiden (s. Abbildung 03). Das Prinzip der CTA beruht auf der Dichteanhebung des Blutes durch intravenöse Gabe von jodhaltigem Kontrastmittel. Dabei wird die Signalstärke von vielen Faktoren beeinflusst; im Wesentlichen von der Menge und Konzentration des Kontrastmittels und von der Hämodynamik. Durch gesteuerte Kontrastmittelapplikation wird das Signal in einem Bereich zwischen 300$400 \mathrm{HE}$ gehalten. 


\subsubsection{CTA-Parameter}

Die CTA wurden auf einem 16-zeiligen Multidetektoren CT (Aquilion ${ }^{\text {TM }}$ TSX-101A; Toshiba, Tokyo, Japan) erstellt. Die Scanparameter sind in den Tabellen 01 und 02 dargelegt.

Die Kontrastmittelinjektion erfolgte mit Immeron ${ }^{\mathrm{TM}} 350(350 \mathrm{mg} \mathrm{Jod} / \mathrm{ml}$, Bracco Diagnostics Inc., Princeton, New Jersey, USA) über einen Nemoto ${ }^{\text {TM }}$ Doppelkolbeninjektor (Nemoto \& Co. Ltd., Tokyo, Japan).

Tabelle 01: CTA-Parameter.

\begin{tabular}{ll}
\hline \hline Parameter & Wert \\
\hline Röhrenspannung & $120 \mathrm{kV}$ \\
Röhrenstrom & $150 \mathrm{mAs}$ \\
Kollimation & $1 \mathrm{~mm}$ \\
Normaler Pitch & 1 \\
Rekonstruierte Schichtdicke & $1 \mathrm{~mm}$ \\
\hline \hline
\end{tabular}

Tabelle 02: Parameter der Kontrastmittelapplikation.

\begin{tabular}{ll}
\hline \hline Parameter KM-Applikation & \\
\hline Konzentration & $350 \mathrm{mg} \mathrm{Jod} / \mathrm{ml}$ \\
Injektionsgeschwindigkeit & $4 \mathrm{ml} / \mathrm{s}$ \\
$\mathrm{NaCl}$ & $30 \mathrm{ml}(4 \mathrm{ml} / \mathrm{s})$ \\
Delay & Art. Bolusdetektion manuell \\
Nadelkaliber & $20 \mathrm{G}$ \\
\hline \hline
\end{tabular}

\subsubsection{Software}

Die CTA wurden auf einer Vitrea 2 Workstation $^{\mathrm{TM}}$ (Vital Images, Inc. Minnetonka, Minnesota, USA.) befundet.

\subsubsection{Bestimmung des Stenosegrades}

Der Grad der Stenose wurde nach drei internationalen Messmethoden bestimmt. Dabei wurde die A. carotis im Verlauf mit sagittaler oder coronarer Projektion auf die 
Karotisbifurkation dargestellt. Die Stenosegrade nach NASCET, ECST und CC wurden auf folgende Weise bestimmt (s. Abbildung 04):
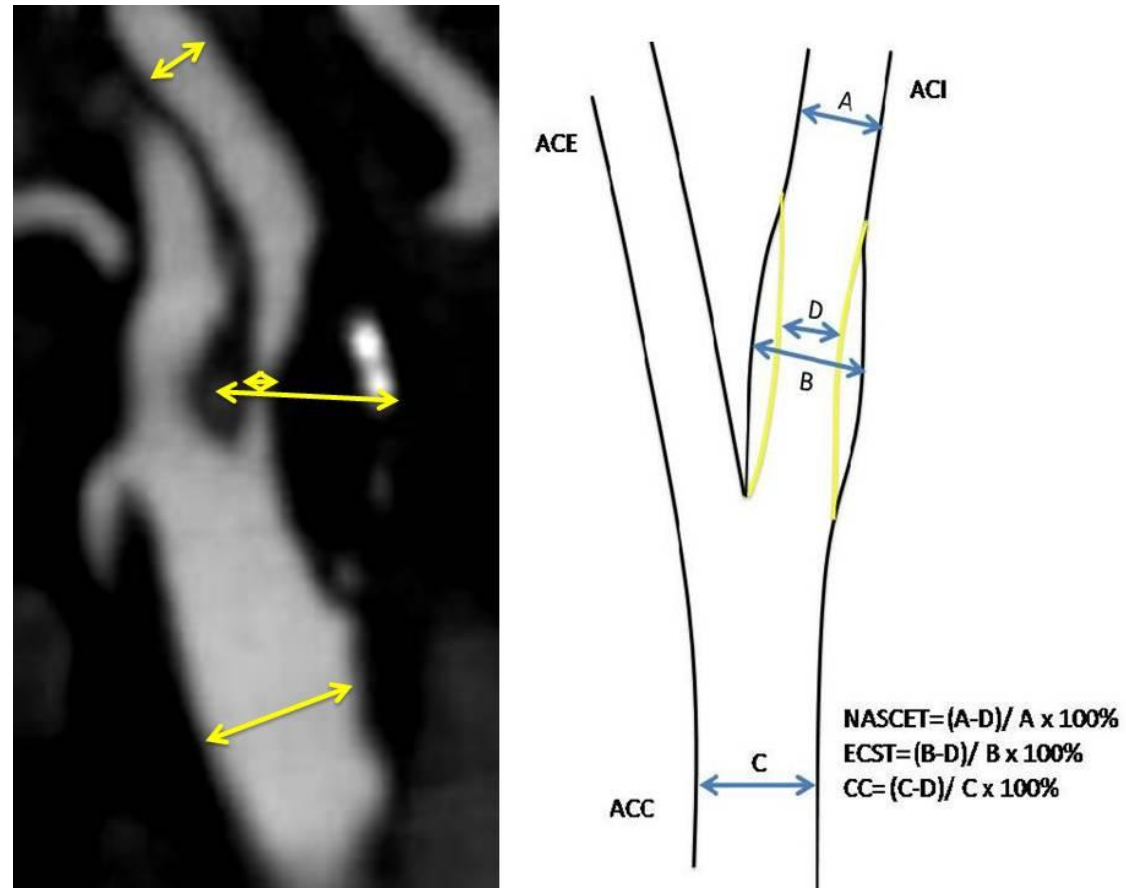

Abbildung 04: Dargestellt sind die Messmethoden für die Stenosegrade nach NASCET, ECST und $\mathrm{CC}$ für die CT-Angiographie. $\mathrm{ACE}=\mathrm{A}$. carotis externa, $\mathrm{ACl}=\mathrm{A}$. carotis interna, $\mathrm{ACC}=\mathrm{A}$. carotis communis. In die ACl wurde mit gelber Farbe eine Plaque skizziert.

\subsubsection{Messung von Weich- und Hartplaque}

Um das Verhältnis von Hartplaque zu Weichplaque zu bestimmen, wurden die Aa. carotis communis und interna in der axialen Projektion dargestellt. Alle Schichten von Beginn der ACC bis einschließlich subpetrösen Teil der $\mathrm{ACl}$ wurden auf Vorhandensein von Stenosen untersucht. Gemessen wurden alle Schichten, die die Stenose abbildeten, d.h. von der Einengung des Lumens bis zur kompletten Rückbildung dieser Einengung. Als Referenz wurde jeweils die Schicht vor der Stenose und nach der Stenose gemessen. Die Schichtdicke betrug $1 \mathrm{~mm}$. Die Schichten wurden in einer zweifachen Vergrößerung bei einer Fensterlage, die der Dichte des Lumens betrug, und einer Fensterbreite von 2 bis 3 Mal der Dichte des Lumens betrachtet. In diesem Bereich konnte sich der Untersucher die Fensterung optimal einstellen. Als Hartplaque wurden alle Anteile der Stenose definiert, die eine höhere Densität als das Lumen aufwiesen, also etwa >300 HE lagen.

Gemessene Parameter waren der äußere Durchmesser des Gefäßes, die Fläche des Gefäßlumens und die Fläche der Hartplaques. Die zu bestimmenden Flächen 
wurden dabei mit dem Cursor abgefahren und von der Software berechnet (s. Abbildung 05). Auf Partialvolumenartefakte wurde Rücksicht genommen, indem das Artefakt mittig abgefahren wurde.

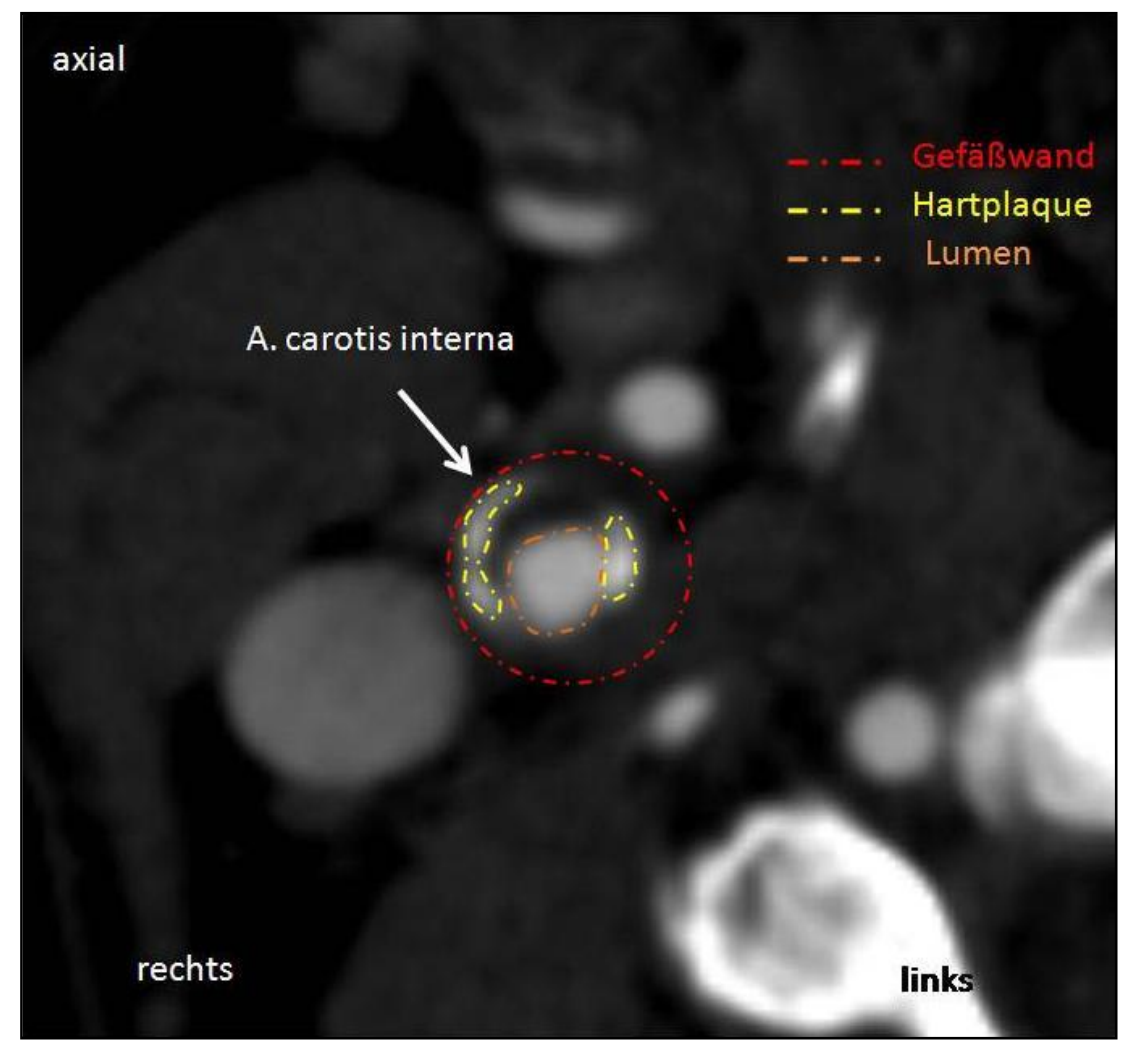

Abbildung 05: Dargestellt ist die Messmethode der Flächen der Gefäßwand (rot), der Hartplaque (gelb) und des Lumens (orange) in der CTA. Dabei wurden die Flächen jeweils mit dem Cursor abgefahren. Die Software bestimmt die

Für jede Schicht wurde die Fläche der Weichplaque bestimmt, indem von der Differenz der Gefäßfläche und der Fläche des Lumens die aus der Dicke errechnete Fläche der Gefäßwand subtrahiert wurde. Dabei wurde jeweils die Wanddicke der ACC auf der letzten Schicht proximal der Stenose und die Wanddicke der ACl auf der ersten Schicht distal der Stenose gemessen. Diesen Schichten galten jeweils als Referenz für den stenosierten Anteil der ACC bzw. ACI. Die Wanddicke wurde wie folgt definiert:

Dicke der Gefäßwand $(\mathrm{mm})=\sqrt{\frac{G e f \text { äß } f l \text { äche }\left(\mathrm{mm}^{2}\right)}{\pi}}-\sqrt{\frac{\operatorname{Lumen}\left(\mathrm{mm}^{2}\right)}{\pi}}$

Die gemessenen bzw. errechneten Flächen wurden zu ihren Volumina addiert: Volumen des Gefäßdurchmessers, Volumen des Lumens, Gesamtplaquevolumen, Gesamthartplaquevolumen und Gesamtweichplaquevolumen. Diese Beziehen sich jeweils auf den stenosierten Bereich. 


\subsection{Klassifizierung der Plaquemorphologie nach Lovett et al.}

Wir klassifizierten die Plaques der Karotisstenosen in der CTA und der DSA von 41 Patienten nach Lovett et al. (2004). 7 Fälle wurden aufgrund geringer Bildqualität der DSA oder CTA ausgeschlossen.

\subsubsection{Lovett- Klassifikation der DSA}

Die Angiographieserien wurden vor Betrachtung der CTA von 2 Untersuchergruppen analysiert. Ausgeschlossen wurden Patienten, die eine Angiographie von schlechter Qualität besaßen.

Zuerst wurde die Plaqueoberfläche beschrieben als ulzeriert, irregulär oder glatt. Plaques wurden als ulzeriert betrachtet, wenn eine der angiographischen Serien auf eine Ulzeration hindeutete. Als irregulär oder glatt wurden sie bezeichnet, wenn es keinen klaren Hinweis für eine Ulzeration gab. Ulzerationen wurden in 4 verschiedene Typen eingeteilt (s. Abbildung 11): Eine Ulzeration, deren Seiten parallel verlaufen oder zu einem Punkt zusammenführen (Typ 1), eine Ulzeration mit schmalem Stiel oder nicht sichtbarem Stiel (Typ 2) und eine Ulzeration, bei der sich das Ende entweder distal (Typ 3) oder proximal (Typ 4) des Hauptteiles verjüngt. Beim Auftreten von mehreren Ulzerationen innerhalb einer Stenose, wurde die größte Ulzeration klassifiziert. Plaques, die keine Ulzerationen aufwiesen, wurden nach 0, 5 oder 6 klassifiziert. Typ 0, wenn sie glatt waren, Typ 5, wenn sie prä- oder poststenotisch dilatiert waren und Typ 6, wenn ihre Gefäßwände irregulär waren oder ein normales Lumen zwischen mehreren glatten Plaques zu sehen war.

\subsubsection{Lovett-Klassifikation der CTA}

Die CTA-Bilder wurden von beiden Untersuchergruppen unabhängig voneinander und geblindet analysiert. Ausgeschlossen wurden CTA mit schlechter Qualität durch Zahnartefakte oder unzureichender Kontrastierung. Die CTA-Bilder wurden dabei in allen drei Projektionen, einmal mit einer Schichtdicke von $1 \mathrm{~mm}$ und ein zweites Mal mit einer maximal intensity projection (MIP) mit einer Extraktion von $4 \mathrm{~mm}$ Datensätzen, betrachtet. Die Fensterung konnte der Betrachter selbst wählen. 
Die Plaques wurden nach den gleichen Typen 0 bis 6 eingeteilt wie in der DSA (s. oben). Sie wurden dabei stets in mehreren parakoronaren, parasagittalen und paraaxialen Schichten betrachtet.

\subsection{Analyse von Mikroembolien durch DWI-MRT}

Es gibt mehrere in der klinischen Praxis verwendete Möglichkeiten zum Nachweis von Mikroembolien. Neurologische Untersuchungen sind allerdings kaum geeignet, da Mikroembolien in der Regel ohne neurologisches Defizit einhergehen. Transkranielle Dopplerunterschungen werden zwar verwendet, doch sie geben keine Aussage über entstandene Läsionen. Eine Korrelation zwischen transkraniellem Doppler und neurologischem Defizit nach SPAC konnte ebenfalls nicht festgestellt werden (Lövblad et al. 2000).

Wir verwenden in dieser Studie die Diffusionsgewichtete Magnetresonanztomographie (DWI-MRT), die eine hohe Sensitivität und Spezifität in der Diagnostik von ischämischen Schlaganfällen besitzt (Fiebach et al. 2002). Der Einsatz der DWI-MRT zur Detektion von mikroembolischen Ereignissen während der SPAC hat sich bereits in zahlreichen Studien bewährt (Asakura et al. 2006, Bendszus und Stoll 2006, Krapf et al. 2006).

\subsubsection{Grundlagen der kraniellen MRT}

Die Magnetresonanztomographie ist der Computertomographie im Nachweis von zerebralen Ischämien überlegen. Dabei wird in der Regel auf eine native MRT zurückgegriffen, die auf T1- und T2- gewichtete Sequenzen basiert. Ein Nachweis erfolgt in den ersten $24 \mathrm{~h}$ nach einem frischen Infarkt durch Veränderung im Blutfluss, morphologischen Veränderungen und Änderung im Signalverhalten. Bereits nach 2-3 h kann der Infarkt durch eine Zunahme der Signalintensität auf T2gewichteten Aufnahmen nachgewiesen werden. Neben der konventionellen MRT kommt den dynamischen MRT-Sequenzen, wie der Diffusion- und Perfusionsgewichtung, eine bedeutende Rolle in der Diagnostik von frühen Ischämien zu. Mithilfe von diffusionsgewichteten Sequenzen lässt sich innerhalb von 30 Minuten ein positiver Befund nachweisen (Fiebach und Schellinger 2003). 


\subsubsection{Diffusionsgewichtete Bildgebung}

Diffusion ist ein passiver physikalischer Prozess, der auf der thermischen Eigenbewegung von Teilchen beruht und zu einer vollständigen Vermischung von Stoffen führt. Dabei spielt für die diffusionsgewichtete Bildgebung der Magnetresonanztomographie nur die Diffusion von Wassermolekülen eine Rolle (Parker 2004). Diese Wassermoleküle befinden sich in festen Gewebestrukturen und können sich so nur in vorgeschriebenen Räumen frei bewegen, da sie immer wieder auf Grenzen oder Hindernisse stoßen, wie Zellmembranen oder Makromoleküle (Bammer 2003). Die Tatsache, dass die Moleküle so in ihrer Diffusion eingeschränkt werden, ist die Grundlage der DWI. Geringe Änderungen in der Ausbreitungsmöglichkeit der Moleküle, wie z.B. eine Einschränkung der Membranpermeabilität oder die Verkleinerung eines interzellulären Raumes, werden durch eine Alteration des diffusionsgewichteten Signals abgebildet. Die Diffusion der Wassermoleküle, die zu einer Signaländerung in der DWI führt, gibt somit eine Auskunft über die Mikrostruktur des Gewebes (Parker 2004).

Diffusionsgewichtete Sequenzen sind T2-gewichtete Sequenzen, in die zwei entgegengesetzte Gradienten mit gleicher Stärke zwischen Hochfrequenzanregung und Datenauslese eingefügt sind (sog. spin-Echo-Sequenzen). Wassermoleküle, die sich entsprechend der Braunschen Molekularbewegung zwischen den Gradientenschaltungen bewegen, führen dazu, dass sich ihre Spins dephasieren und ein niedriges Bildsignal entsteht. Die Differenz aus der Signalintensität einer T2gewichteten Aufnahme und diesem Signalverlust ergibt die Signalintensität eines Voxels in der DWI (Fiebach und Schellinger 2003). Ist die Molekularbewegung nicht eingeschränkt, führt dies zu einem Signalverlust im gesamten Bild. Bei eingeschränkter Diffusion werden zwischen den Gradientenschaltungen mehr Protonenspins rephasiert. Daraus erfolgt ein geringerer Signalverlust (Bammer 2003). Da wir nicht freie Diffusion von Wasser, sondern die Diffusion von Wassermolekülen in einem vorgeschriebenen Raum betrachten, die von Konzentrations-, Druck-, Temperatur- und osmotischen Gradienten abhängig ist, spricht man nicht vom Diffusionskoeffizienten, sondern vom "scheinbaren“, apparenten Diffusionskoeffizienten (ADC) (Cercignani und Horsfield 2001). 


\subsubsection{2. b-Wert und Infarktdarstellung}

Die Signalintensität hängt außer vom Diffusionskoeffizienten von der Diffusionssensitivität der Pulssequenz ab. Diese wird mit dem b-Wert (gemessen in $\mathrm{s} / \mathrm{mm}^{2}$ ) bezeichnet und hängt ab von der Amplitude der diffusionsgewichteten Gradienten, der Schaltdauer der Gradienten und der Diffusionszeit. Zur Berechnung des ADC sind unterschiedliche diffusionsgewichtete Bilder, d.h. Aufnahmen mit verschiedenen b-Werten notwendig (Fiebach und Schellinger 2003). Hierbei darf sich der Patient zwischen den Messungen nicht bewegen. Nach Stejskal und Tanner (1965) berechnet man den ADC-Wert aus der Geradensteigung, die sich ergibt, indem man den Logarithmus der Signale aus mehreren unterschiedlich stark diffusionsgewichteten Bildern über den b-Werten aufträgt. Die ADC-Map ist die ortsaufgelöste Darstellung der rechnerisch ermittelten ADC-Werte. Diffusionseingeschränkte Areale sind auf ihr hell dargestellt, also hyperintens und diffusionserleichterte Areal dunkel, also hypointens. Hyperintensität entsteht auch bei Geweben mit langer T2-Relaxationszeit, sog. T2-shine-trough (Fiebach und Schellinger 2003). Zur Berechnung der ADC-map werden hierbei ADC-Bilder verwendet, die nicht durch Änderungen der T2-Relaxationszeit gestört sind. Die Qualität steigt mit der zur Berechnung einbezogenen Zahl von diffusionsgewichteten Bildern (Bammer 2003).

Akute (<24 h) und frühe subakute (1 bis 7 Tage) Infarkte erscheinen hyperintens in der DWI-Bildgebung. Hyperakute $(<6 \mathrm{~h})$ Infarkte erscheinen ebenfalls hyperintens in der DWI-Bildgebung, aber isointens auf T2-gewichteten Bildern. Erst nach etwa einer Woche (spätakut) verlieren sie ihre Auffälligkeiten in der DWI, sind aber in der T2gewichteten Bildgebung durch Hyperintensität als ischämisches Ereignis erkennbar.

\subsubsection{MRT-Parameter}

Untersucht wurde auf einem Siemens Magnetom Tim-Trio System (3 Tesla) mit der Software syngo MR B15 ${ }^{\mathrm{TM}}$ (Siemens Medical Solutions, Erlangen, Deutschland) mit einer Siemens-12-Kanal Kopfspule (Siemens Medical Solutions, Erlangen, Deutschland). Es wurden jeweils neben einem localizer eine Diffusionsgewichtung (DWI), eine suszeptibilitätsgewichtete Bildgebung (SWI) und eine fluid attenuated inversion recovery (FLAIR) angefertigt (s. Tabelle 03). 
Tabelle 03: MRT-Parameter.

\begin{tabular}{lllll}
\hline Parameter & localizer & DWI & SWI & FLAIR \\
\hline TR & $20 \mathrm{~ms}$ & $4800 \mathrm{~ms}$ & $38 \mathrm{~ms}$ & $9000 \mathrm{~ms}$ \\
TE & $5 \mathrm{~ms}$ & $150 \mathrm{~ms}$ & $20 \mathrm{~ms}$ & $85 \mathrm{~ms}$ \\
Schichtdicke & $10 \mathrm{~mm}$ & $6 \mathrm{~mm}$ & $3 \mathrm{~mm}$ & $6 \mathrm{~mm}$ \\
Schichtabstand & - & $10 \%$ & $20 \%$ & $10 \%$ \\
Schichtanzahl & 1 & 20 & 40 & 20 \\
Flip-Winkel & $40^{\circ}$ & - & $15^{\circ}$ & $150^{\circ}$ \\
Untersuchungszeit & $9 \mathrm{~s}$ & $83 \mathrm{~s}$ & $174 \mathrm{~s}$ & $200 \mathrm{~s}$ \\
\hline
\end{tabular}

\subsubsection{Software}

Die MRT-Bilder wurden auf einer Leonardo Workstation $^{\mathrm{TM}}$ (Siemens Medical Solutions, Erlangen, Deutschland) befundet.

\subsubsection{Messung der DWI-Läsionen}

6 mm-Schichten der prä- und postinterventionellen kraniellen MRT wurden verglichen. Akute ischämische Läsionen wurden diagnostiziert, wenn sie sich in der axialen Schicht der B1000-Karte der Diffusionsgewichtung als Fokus mit hoher Signalintensität dargestellt haben. Zur Sicherung der Diagnose einer ischämischen Läsion wurde der Focus in der FLAIR-Bildgebung betrachtet. Hier sollte sich eine akute diffusionseingeschränkte ischämische Läsion noch nicht darstellen. Ältere Ischämien hingegen sind hier aufgrund der Ausbildung eines vasogenen Ödems als Fokus mit hoher Signalintensität sichtbar (Gauvrit et al. 2006). Die Fläche der ischämischen Läsionen wurde durch die Software berechnet, indem die Ränder mit dem Cursor abgefahren wurden (s. Abbildung 06).

Jede ischämische Läsion wurde außerdem hinsichtlich ihrer Lokalisation eingeteilt. Die Lokalisation bezog sich auf die Hemisphäre (ipsilateral, kontralateral), das arterielle Versorgungsgebiet (ACA, ACM, ACP), auf die Höhe in Bezug auf die Seitenventrikel (upper, middle, lower), auf die Lokalisation innerhalb einer axialen Schicht (kortikal, weiße Substanz oder tiefe graue Substanz) und auf den Lappen bzw. Anteil des Gehirns (Frontallappen, Parietallappen, Temporallappen, Okzipitallappen, Basalganglien, Thalamus, infratentoriell). Große ischämische 
Läsionen über $1 \mathrm{~cm}^{2}$ wurden als Infarkte dokumentiert. Kleiner als $1 \mathrm{~cm}^{2}$ große Läsionen wurden als DWI-Läsion bzw. Mikroemboli bezeichnet. Alte ischämische Läsionen, d.h. Läsionen, die bereits auf dem präinterventionellen MRT sichtbar waren, wurden getrennt von neuen ischämischen Läsionen dokumentiert.

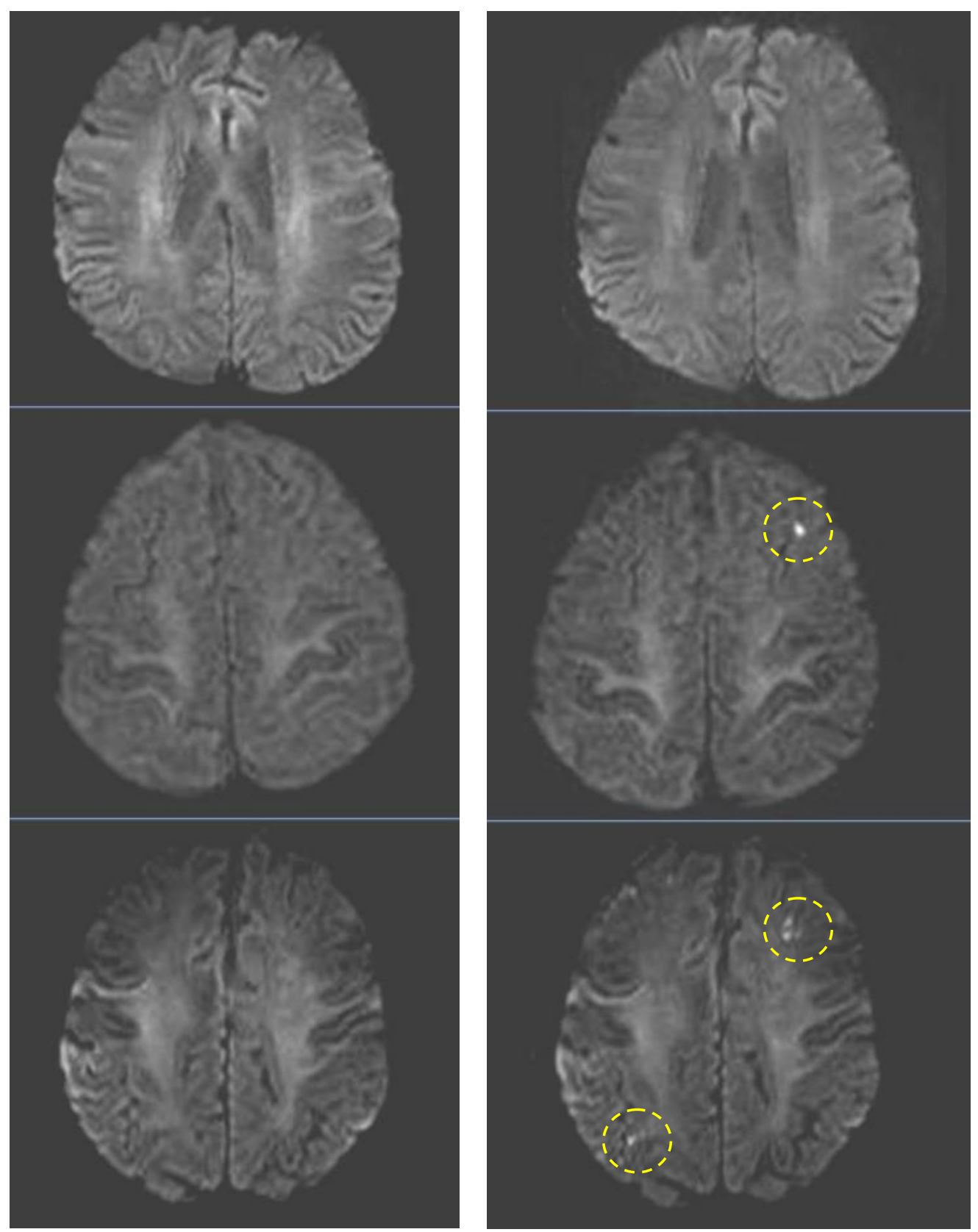

Abbildung 06: Abgebildet sind diffusionsgewichtete MRT-Sequenzen vor SPAC (links) und nach SPAC (rechts). Die MRT-Bilder in der Mitte rechts und unten rechts nach der Intervention zeigen mikroembolische Infarkte (als Hyperintensitäten zu erkennen). 


\subsection{Statistische Auswertung}

Für die statistische Auswertung wurde das Programm Statistical Package for the Social Sciences ${ }^{\mathrm{TM}}$ (Version 11.5, SPSS, Chicago, IL, USA) verwendet.

Im Rahmen der deskriptiven Statistik wurden, soweit möglich, folgende Kenngrößen ermittelt: Häufigkeit, Mittelwert, Median, Varianz, Standardabweichung, kleinster und größter Wert.

Interferenzstatistisch kamen bei nominalen Daten für normalverteilte Daten der tTest, und der Mann-Whitney-U-Test für nicht normalverteilte Daten zur Anwendung. Für kategoriale Daten wurden der zweiseitige Chi-Quadrat-Test und der exakte Test nach Fisher bei Häufigkeiten der Einzelwerte unter 5 verwendet. Neben einer optischen Überprüfung wurde zur Prüfung der Verteilungsform der KolmogorovSmirnov-Test eingesetzt. Bei Subgruppenanalysen wurden für Risikofaktoren Odds Ratios berechnet. Als Signifikanzniveau wurde ein $p$-Wert von kleiner 0,05 festgelegt, entsprechend einer Irrtumswahrscheinlichkeit von kleiner 5\%. Hochsignifikant waren Ergebnisse mit einem Signifikanzniveau von $p=0,01$, also einer Irrtumswahrscheinlichkeit von unter $1 \%$. Des Weiteren erfolgten Korrelationsanalysen nach Pearson zur Beurteilung des Zusammenhangs zwischen verschiedenen metrischen Parametern aus CTA oder DSA und Anzahl und Fläche der neuentstandenen Läsionen in der DWI-MRT. 


\section{$\underline{\text { 5. Ergebnisse }}$}

\section{$\underline{\text { 5.1. Deskriptive Statistik }}$}

Die demografischen Daten der eingeschlossenen Patienten sind in Tabelle 04 wiedergegeben. In die Studie wurden 46 Patienten eingeschlossen, davon 36 Männer (78,26\%) und 10 Frauen (21,74\%). 26 Patienten (56,52\%) wurden an der linken A. carotis behandelt, $18(39,13 \%)$ an der rechten und $2(4,35 \%)$ an beiden Karotiden. Das mittlere Alter der Patienten lag bei 67,39 Jahren (min. 49; max. 83). Die Stenosegrade nach NASCET lagen im Mittel bei 67,78\% (min. 34,29\%; max. $88,89 \%)$. In 34 Fällen $(70,83 \%)$ war die Stenose symptomatisch, in 14 Fällen $(29,17 \%)$ asymptomatisch. In allen Fällen konnte die stenosierte Arterie rekanalisiert werden. Bei 3 Patienten traten nach Intervention klinisch manifeste Komplikationen auf, darunter 1 TIA, 1 minor stroke und 1 major stroke mit Todesfolge. Von diesen 3 Patienten hatten 2 eine symptomatische Stenose und der dritte einen kontralateralen Verschluss der ACl. Nur einer dieser Patienten wurde einer SPAC mit zerebraler Protektion zugeführt. Insgesamt wurden 17 Patienten (35,4\%) einer SPAC mit zerebraler Protektion zugeführt (s. Tabelle 05). Der Patient, der einen major stroke erlitt, hatte keine zerebrale Protektion während der SPAC erhalten. Die klinische Komplikationsrate liegt insgesamt bei 6,25\%.

Tabelle 04: Demografische Daten der eingeschlossenen Patienten.

\begin{tabular}{lll}
\hline \hline & & $\mathbf{N}$ \\
\hline Geschlecht & männlich & $10(21,74 \%)$ \\
& weiblich & $36(78,26 \%)$ \\
Seite & & \\
& rechts & $18(39,13 \%)$ \\
& links & $26(56,52 \%)$ \\
Symptomatische Stenose & beidseits & $2(4,35 \%)$ \\
Verschluss kontralateral & & $34(70,83 \%)$ \\
Statintherapie & & $8(16,67 \%)$ \\
CRP erhöht & & $25(52,08 \%)$ \\
Fettstoffwechselstörung & & $13(27,08 \%)^{*}$ \\
\hline
\end{tabular}


Tabelle 05: Technische Daten zur SPAC.

$\mathbf{N}$

\begin{tabular}{lll}
\hline Stentsystem & & \\
& BSCW & $42(87,5 \%)$ \\
& CPRXNSS & $3(6,25 \%)$ \\
Protektionssystem & SCCRXS & $3(6,25 \%)$ \\
Entlüftung & & $17(35,42 \%)$ \\
\hline \hline
\end{tabular}

$\overline{\overline{B S C W}=\text { Boston Scientific Wallstent }{ }^{\mathrm{TM}} \text {, CPRXNSS= Cordis Precise RX Nitinol Stent System }{ }^{\mathrm{TM}} \text {, SCCRXS= Sinus Carotid Conical }}$ RX Stent ${ }^{\mathrm{TM}}$

Tabelle 06: Klinische Risikofaktoren für periinterventionelle Mikroembolien in DWI-MRT.

\begin{tabular}{lllll}
\hline \hline & $\begin{array}{l}\text { Keine neuen } \\
\text { DWI-Läsionen }\end{array}$ & $\begin{array}{l}\text { Neue DWI- } \\
\text { Läsionen }\end{array}$ & Odds Ratio & p-Wert \\
\hline $\mathbf{n}$ & $17(35,42 \%)$ & $31(64,58 \%)$ & - & - \\
Mittleres Alter & 65,29 & 68,42 & - & 0,187 \\
Alter > 70Jahre & $4(8,33 \%)$ & $12(25 \%)$ & 2,05 & 0,286 \\
Männlich & $11(22,92 \%)$ & $26(54,42 \%)$ & 2,84 & 0,163 \\
Rechte Karotis & $4(8,33 \%)$ & $16(33,33 \%)$ & 3,47 & $0,059^{*}$ \\
Symptomatisch & $14(29,17 \%)$ & $20(41,67 \%)$ & 0,39 & 0,194 \\
Kontralateraler & $3(6,25 \%)$ & $5(10,42 \%)$ & 1,114 & 1,000 \\
Verschluss & & & & 0,678 \\
Mittlerer & $68,86 \%$ & $67,18 \%$ & - & \\
Stenosegrad & & & & 0,93 \\
nach NASCET & & $16(33,33 \%)$ & 0,95 & 0,324 \\
Statintherapie & $9(18,75 \%)$ & $7(14,58 \%)$ & 0,5 & \\
CRP erhöht & $6(1,25 \%)$ & &
\end{tabular}

In 31 Fällen (64,58\%) konnte nach SPAC mindestens eine neue Läsion in der DWIMRT beobachtet werden (s. Tabelle 06). Dabei lag der Mittelwert von neuen Läsionen bei 3,65 (min. 0; max. 19). Die mittlere Gesamtfläche von neuen Läsionen lag bei $0,7 \mathrm{~cm}^{2}$ (min. 0; max. 6,99). Bei 14 Patienten (29,17\%) traten Läsionen außerhalb des Stromgebietes der behandelten Arterie auf.

In Tabelle 06 sind klinische Risikofaktoren für das Auftreten von mikroembolischen Ereignissen nach SPAC dargestellt. Männliches Geschlecht, asymptomatische Stenosen und Stenosen der rechten A. carotis waren bei Patienten mit neuen DWI- 
Läsionen nach der SPAC häufiger vertreten. Keiner dieser Risikofaktoren war signifikant. Eine Stenose der rechten Seite erhöhte die Wahrscheinlichkeit von Läsionen nach Intervention mit einer Odds Ratio von 3,47 und ist mit einem p-Wert von 0,059 fast signifikant. Ebenso zeigt sich, dass das mittlere Alter der Patienten mit 68,42 Jahre gegenüber 65,29 Jahren in der Gruppe der Patienten mit neuen Läsionen nach SPAC etwas höher lag ( $p$-Wert= 0,187 ). In dieser Gruppe waren mit 12 auch $3 \mathrm{Mal}$ mehr Patienten über 70 Jahren vertreten. Daraus ergab sich für den Risikofaktor Alter über 70 Jahren eine Odds Ratio von 2,05, welche mit einem p-Wert von 0,286 nicht signifikant ist.

\subsection{Zusammenhang Plaquezusammensetzung und DWI-Läsionen}

Tabelle 07 zeigt die allgemeinen Messdaten der CT-Angiographie. Der Weichplaqueanteil an der Gesamtplaque lag im Mittel bei 73,41\% (min. 20,86\%; max. 100\%) und die Anzahl der Hartplaques bzw. Hartplaquespangen bei 4,21 (min. 0; max. 16). Zwischen dem Weichplaqueanteil und neuen DWI-Läsionen nach SPAC konnte ein statistischer Zusammenhang festgestellt werden. Der Weichplaqueanteil korrelierte positiv mit der Zahl neuer DWI-Läsionen nach SPAC (s. Tabelle 08). Im Punktdiagramm in Abbildung 07 wird dieser Trend verdeutlicht. Mit einem $p$-Wert von 0,094 ist dieser Trend nicht signifikant. Allerdings korreliert der Weichplaqueanteil signifikant mit der Fläche dieser neuentstandenen DWI-Läsionen ( $p$-Wert= 0,045; s. Tabelle 08). Diese signifikante Korrelation wird in Abbildung 08 als Punktdiagramm dargestellt.

Eine Boxplotanalyse in Abbildung 09, jeweils für Patienten mit und ohne neue DWILäsionen, zeigt, dass der Weichplaqueanteil im Mittel bei Patienten mit neuen Läsionen höher lag als bei Patienten ohne neue Läsionen (65,91\% vs. 77,53\%).

Der Vergleich der Anzahl an Hartplaques innerhalb der Stenose mit dem Auftreten neuer DWI-Läsionen, bzw. mit der Fläche neuer Läsionen, zeigt einen negativen Trend ( $p$-Wert=0,164 bzw. 0,084) (s. Tabelle 08). 
Tabelle 07: Allgemeine Messdaten der CTA.

\begin{tabular}{llccccc}
\hline \hline & & N & Minimum & Maximum & Mittelwert & $\begin{array}{l}\text { Standard- } \\
\text { abweichung }\end{array}$ \\
$\begin{array}{l}\text { Stenose- } \\
\text { grad nach }\end{array}$ & & & & & & \\
& NASCET & 48 & $34,29 \%$ & $88,89 \%$ & $67,78 \%$ & $13,25 \%$ \\
& ECST & 48 & $70,11 \%$ & $96,08 \%$ & $85,49 \%$ & $6,96 \%$ \\
\hline $\begin{array}{l}\text { Anteil } \\
\text { Weichplaque }\end{array}$ & CC & 48 & $61,73 \%$ & $92,98 \%$ & $81,85 \%$ & $7,73 \%$ \\
\hline $\begin{array}{l}\text { Anzahl } \\
\text { Hartplaques }\end{array}$ & 48 & $20,86 \%$ & $100 \%$ & $73,41 \%$ & $22,38 \%$ \\
\hline \hline
\end{tabular}

Tabelle 08: Korrelationen nach Pearson von Weichplaqueanteil zu Anzahl und Fläche neuer DWILäsionen.

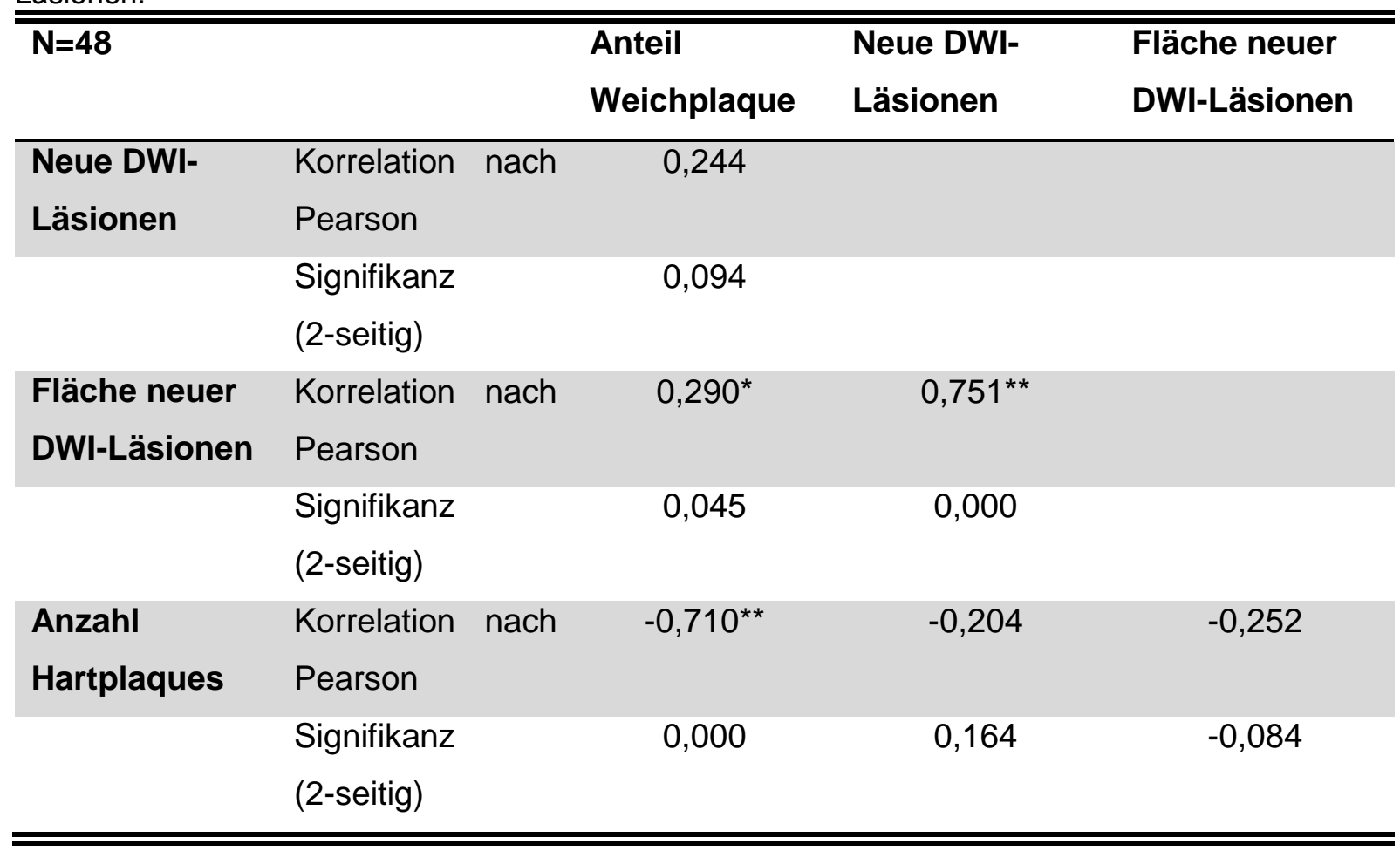




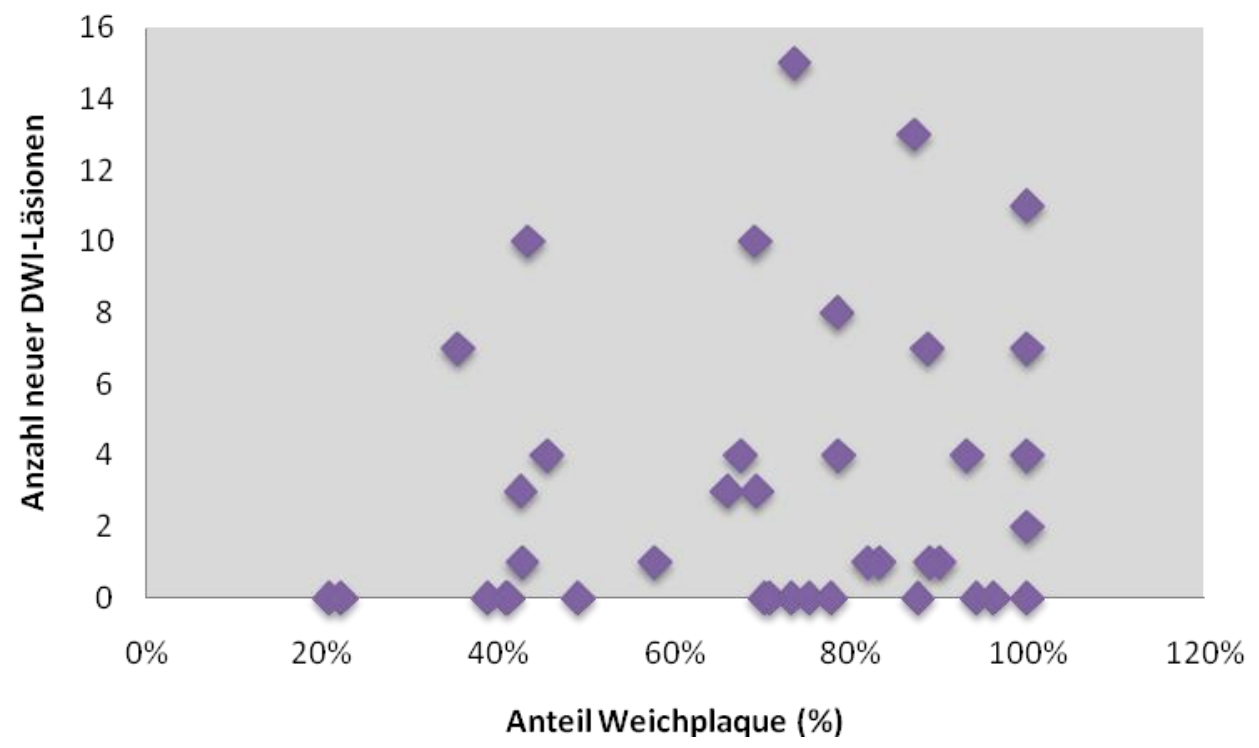

Abbildung 07: Punktdiagramm. Korrelation zwischen Weichplaqueanteil zu Anzahl neuentstandener DWI-Läsionen nach Intervention. Das Diagramm zeigt eine deutlich positive Korrelation der beiden Parameter (nicht signifikant).

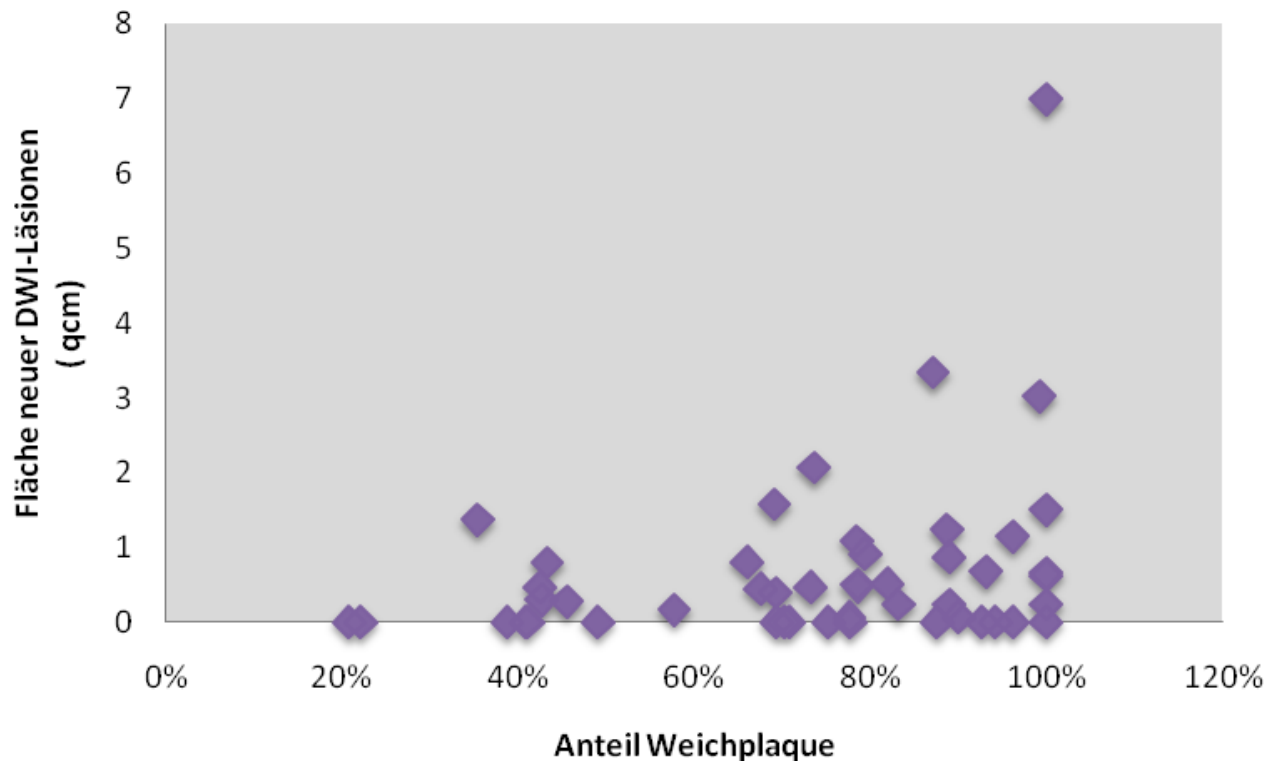

Abbildung 08: Punktdiagramm. Korrelation zwischen Weichplaqueanteil und Fläche neuentstandener DWI-Läsionen nach Intervention. Das Diagramm zeigt eine deutlich positive Korrelation der beiden Parameter. In Tabelle 09 wird gezeigt, dass diese Korrelation mit $\mathrm{p}=0,045$ signifikant ist. 


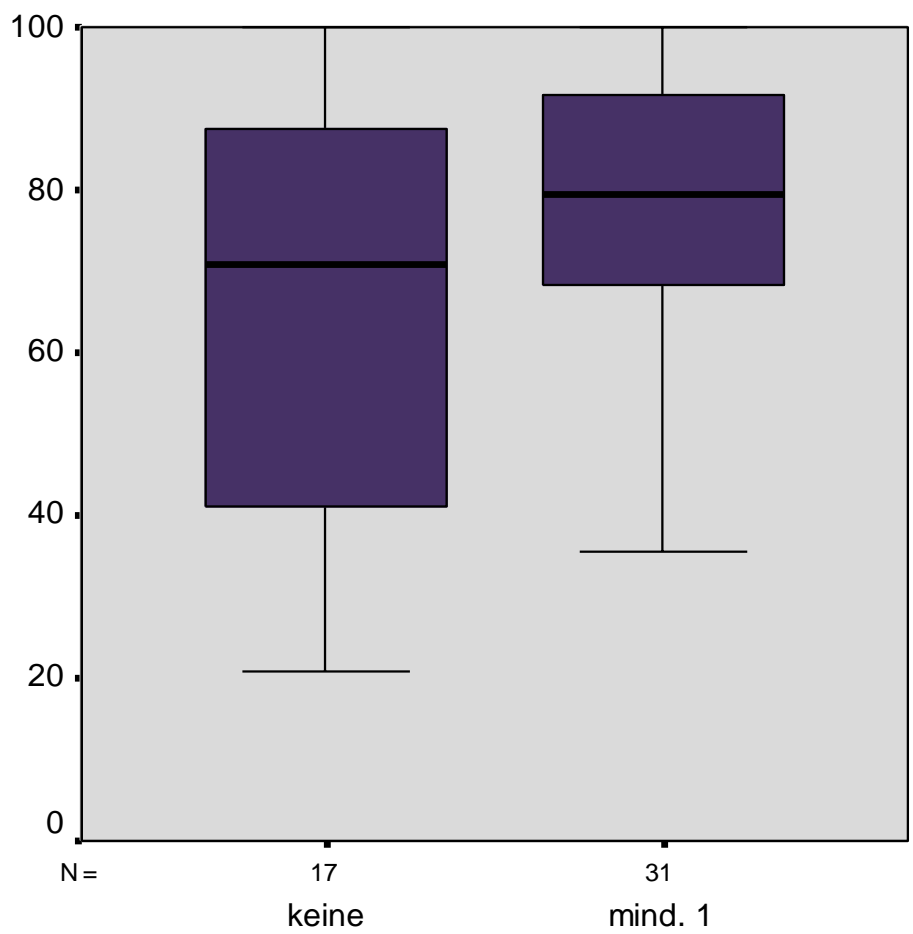

Neue DWI-Läsionen

Abbildung 09: Boxplot. Weichplaqueanteil der Plaques für jeweils die Gruppe der Patienten mit neuen Läsionen nach Intervention und für die Gruppe ohne Läsionen. Das Diagramm zeigt, dass Patienten mit neuen DWI-Läsionen einen höheren Weichplaqueanteil haben.

\subsection{Zusammenhang Ulzerationen und DWI-Läsionen}

Es konnte gezeigt werden, dass Patienten mit ulzerierten Plaques mehr neue DWILäsionen nach SPAC hatten als solche ohne ulzerierte Plaques. Die Boxplot-Analyse in Abbildung 10 zeigt einen Median bei Ulzeration von 3 Läsionen gegenüber 1 Läsion bei nicht ulzerierten Plaques $(\mathrm{OR}=1,94)$. 


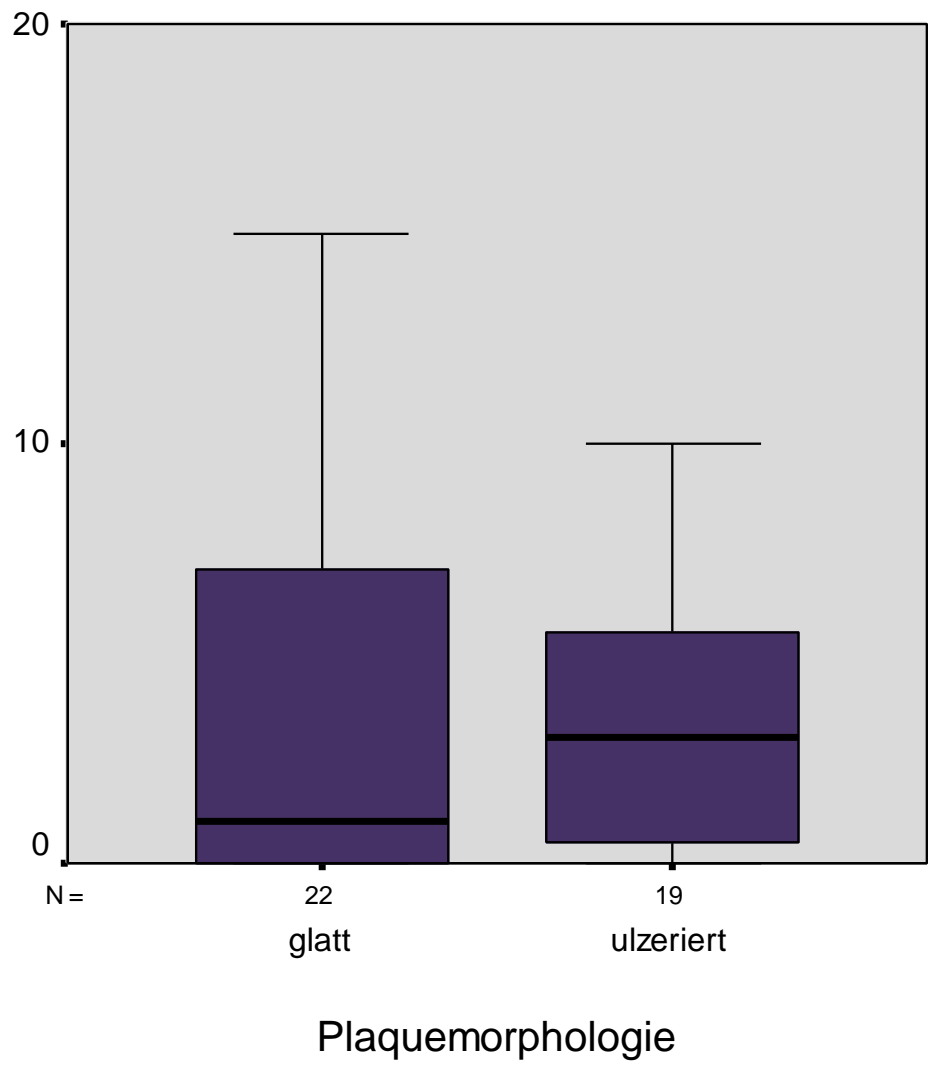

Abbildung 10: Boxplot. Dargestellt ist die Anzahl neuer DWI-Läsionen jeweils für die Gruppen der Patienten mit ulzerierten Plaques in der DSA und für die Gruppe mit glatten Plaques.

\subsection{Andere Risikofaktoren anhand der CTA}

Die Tabelle 09 zeigt die Korrelation der Stenosegrade nach NASCET, ECST und CC zu der Anzahl und Fläche neuentstandener DWI-Läsionen. Es konnte nicht gezeigt werden, dass Stenosegrade und DWI-Läsionen nach SPAC miteinander korrelieren. Der Vergleich der Stenosegrade, die nach den verschiedenen Methoden, NASCET, ECST und CC gemessen wurden, zeigt, dass alle hoch-signifikant miteinander korrelieren $(p-W e r t<0,001)$. 
Tabelle 09: Korrelationen nach Pearson von Stenosegraden nach NASCET, ECST und CC zu neuentstandenen DWI-Läsionen nach Intervention

\begin{tabular}{|c|c|c|c|c|c|}
\hline \multirow[t]{2}{*}{$\mathrm{N}=48$} & \multicolumn{4}{|l|}{ Stenose-grade } & \multirow{2}{*}{$\begin{array}{l}\text { Neue DWI- } \\
\text { Läsionen }\end{array}$} \\
\hline & nach: & NASCET & ECST & CC & \\
\hline \multirow{3}{*}{$\begin{array}{l}\text { Stenose-grad } \\
\text { nach: ECST }\end{array}$} & Korrelation nach & $0,820^{\star *}$ & & & \\
\hline & Pearson & & & & \\
\hline & $\begin{array}{l}\text { Signifikanz } \\
\text { (2-seitig) }\end{array}$ & 0,000 & & & \\
\hline \multirow[t]{3}{*}{ CC } & Korrelation nach & $0,886^{* *}$ & $0,875^{* *}$ & & \\
\hline & Pearson & & & & \\
\hline & $\begin{array}{l}\text { Signifikanz } \\
\text { (2-seitig) }\end{array}$ & 0,000 & 0,000 & & \\
\hline \multirow{3}{*}{$\begin{array}{l}\text { Neue DWI- } \\
\text { Läsionen }\end{array}$} & Korrelation nach & 0,135 & 0,086 & 0,066 & \\
\hline & Pearson & & & & \\
\hline & $\begin{array}{l}\text { Signifikanz } \\
\text { (2-seitig) }\end{array}$ & 0,359 & 0,560 & 0,657 & \\
\hline \multirow[t]{2}{*}{$\begin{array}{l}\text { Fläche neuer } \\
\text { DWI-Läsionen }\end{array}$} & $\begin{array}{l}\text { Korrelation nach } \\
\text { Pearson }\end{array}$ & 0,039 & $-0,065$ & 0,006 & $0,751^{* *}$ \\
\hline & $\begin{array}{l}\text { Signifikanz } \\
\text { (2-seitig) }\end{array}$ & 0,528 & 0,662 & 0,970 & 0,000 \\
\hline
\end{tabular}

** Die Korrelation ist auf dem Niveau von 0,01 (2-seitig) signifikant.

\subsection{Klassifikation der Plaquemorphologie nach Lovett et al.}

In unserer Studie wurden 41 Karotisstenosen nach ihrer Plaquemorphologie klassifiziert (siehe 4.4.). 7 der 48 Fälle konnten aufgrund schlechter Bildqualität der CTA oder DSA nicht klassifiziert werden. Die Abbildung 11 zeigt die CTA und DSA Bilder der Stenosen für jede Gruppe im Vergleich. 


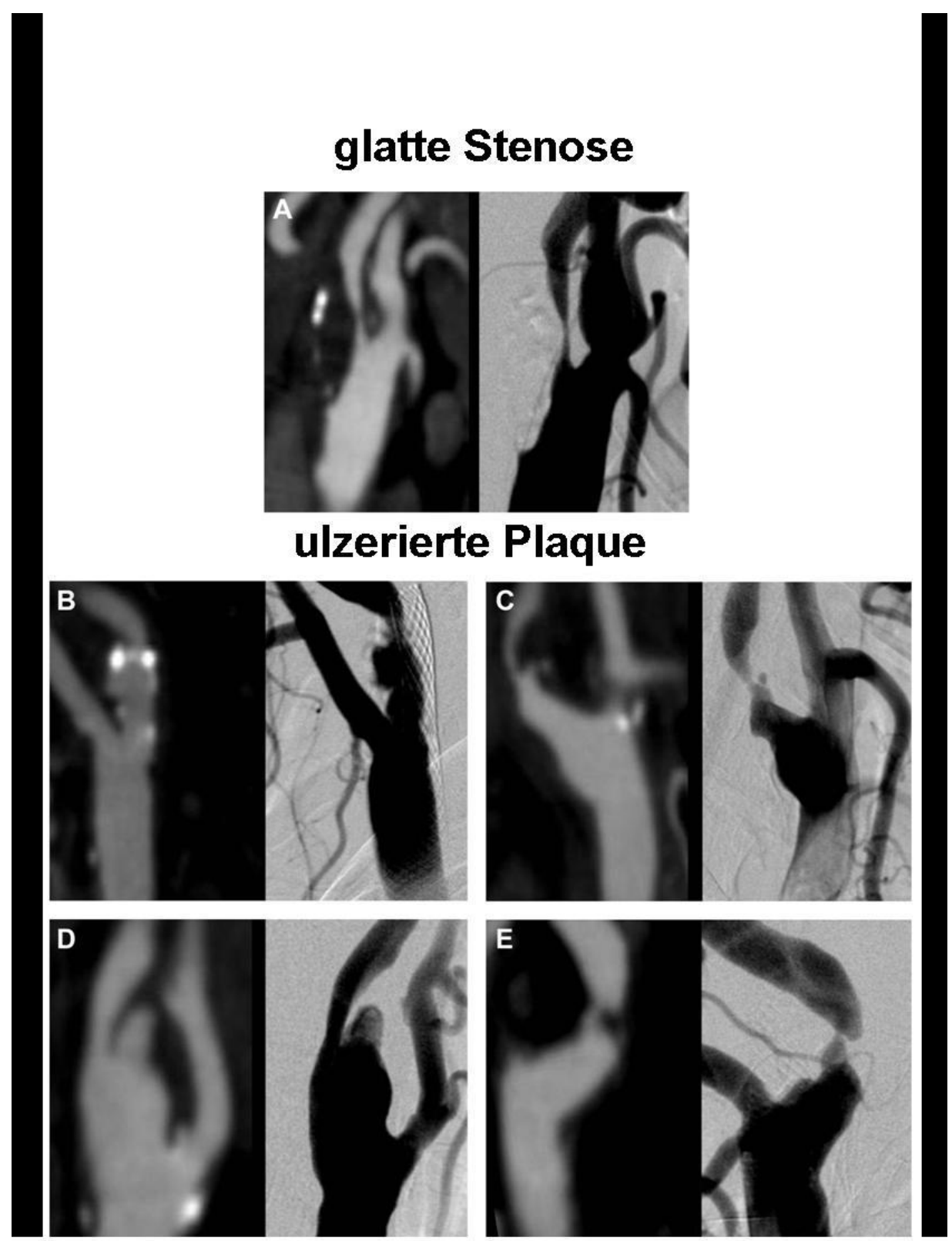

\section{unregelmäßige Plaqueoberfläche}
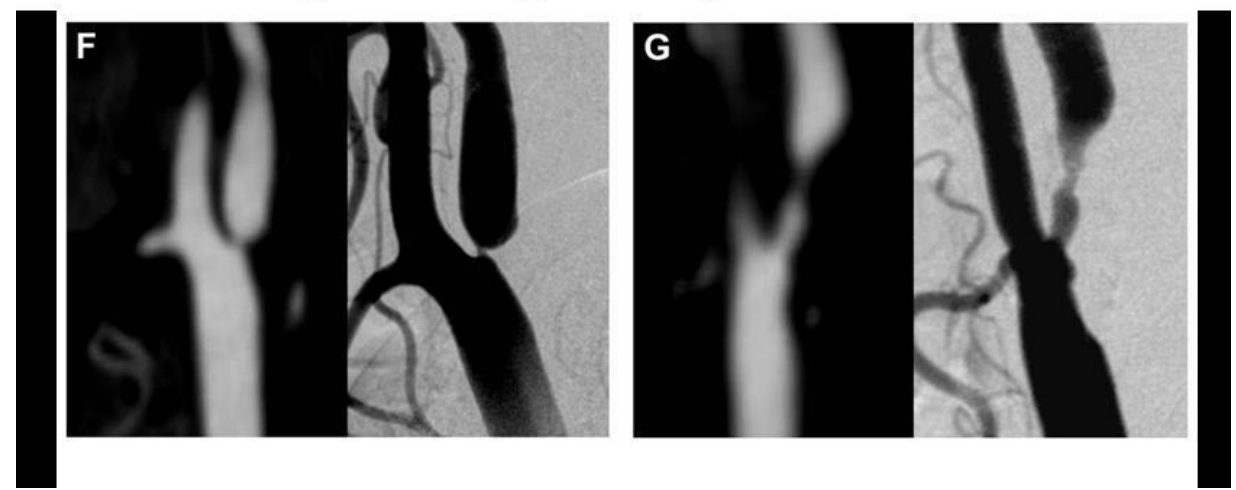

Abbildung 11: Dargestellt sind alle 7 Typen der Plaquemorphologie nach der Klassifikation von Lovett et al. (2004). Links ist jeweils die Stenose in der CTA dargestellt und rechts in der DSA. A: Keine Ulzeration, glatte Gefäßwand (Typ 0), B: Gerade Ulzeration mit parallelen oder spitzaufeinander zulaufenden Seiten (Typ 1), C: Ulzeration mit schmalem oder nicht sichtbarem Hals (Typ 2), D: Nach distal sich verjüngende Ulzeration (Typ 3), E: Nach proximal sich verjüngende Ulzeration (Typ 4), F: Keine Ulzeration, irregulärer Gefäßverlauf, prä- oder poststenotische Dilatation (Typ 5), G: Keine Ulzeration, irregulärer Gefäßverlauf zwischen mehreren Plaques (Typ 6). 


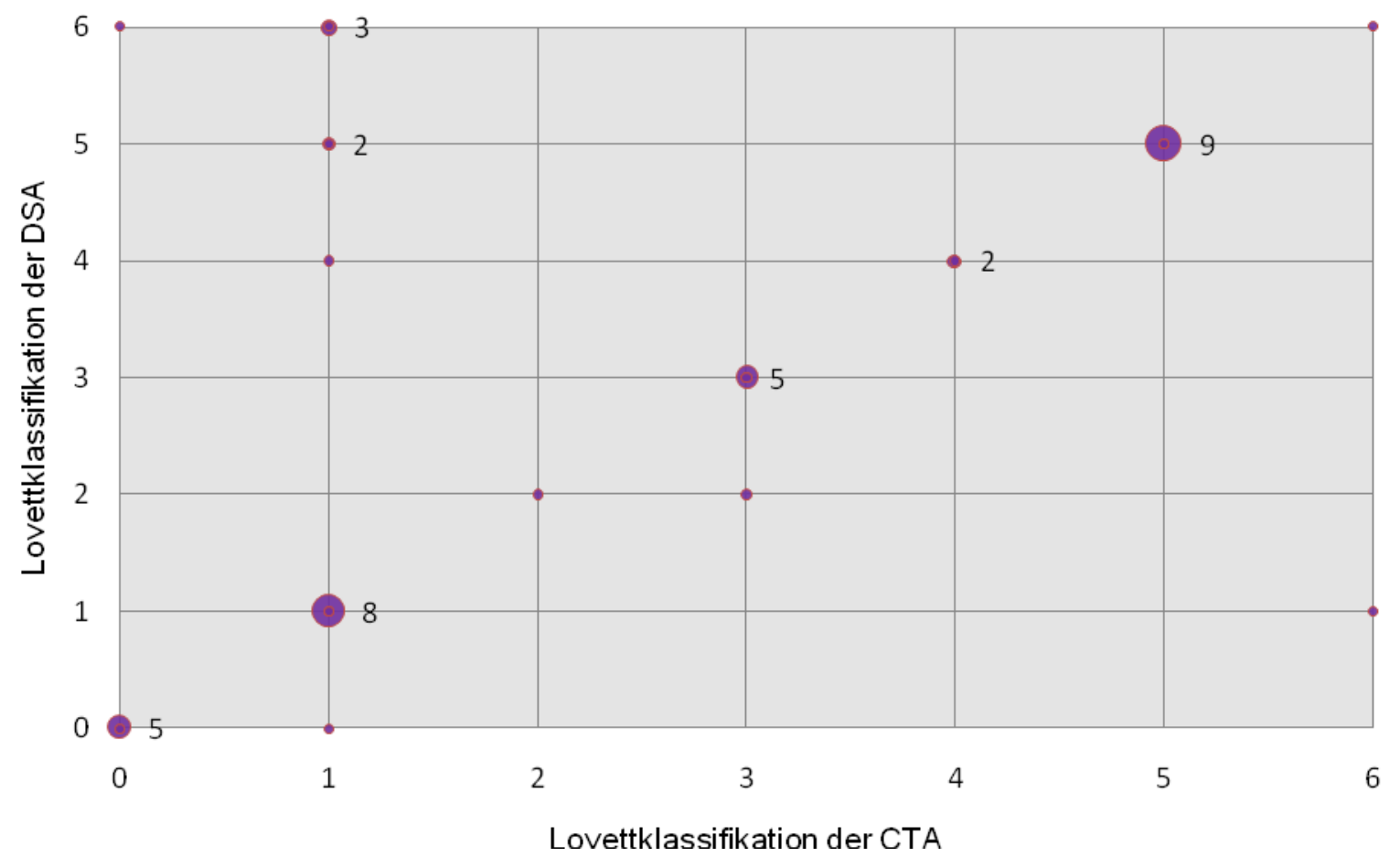

Abbildung 12: Dargestellt sind für jeden einzelnen Fall die Klassifikation der Plaque nach Lovett et al. in der CTA und der DSA. Jeder kleine Kreis repräsentiert einen Fall; große Kreise mehrere Fälle. Die Zahl neben dem Kreis steht für die Anzahl der Fälle.

Die Abbildung 12 zeigt die Einteilungen der Plaquemorphologie für jeden Fall jeweils in der DSA und CTA. Dabei zeigen die Abbildungen 11 und 12, dass sich diese Klassifikation nach Lovett et al. in beiden Verfahren sehr gut reproduzieren lässt. In 31 von 41 Fällen stimmen die Klassifizierungen in der CTA und DSA überein. Teilt man die 6 verschiedenen Typen in die Subgruppen Plaque mit Ulzeration und Plaque ohne Ulzeration auf, so stimmt die DSA mit der CTA in 34 von 41 Fällen überein. Nimmt man die DSA als Goldstandard in der Bestimmung von Plaqueulzerationen bei Karotisstenosen, so zeigt Tabelle 10, dass die CTA eine Sensitivität von 94,7\% bei einer Spezifität von $72,7 \%$ in dieser Fragestellung hat.

Parallel dazu wurde in unserer Studie der Einfluss der verschiedenen Typen von 0 bis 6 der Klassifikation nach Lovett et al. auf das Auftreten von DWI-Läsionen nach SPAC untersucht (s. Abbildungen 13 und 14). Es konnte sowohl in der CTA als auch in der DSA gezeigt werden, dass die Typen 1, 2, 3 und 4 im Median mehr DWILäsionen haben als die Typen 0,5 und 6. Am meisten Läsionen im Median hatten die Typen 1 und 4. Dabei zeigte die Klassifikation der Plaquemorphologie in der CTA für Typ $1 \mathrm{im} \mathrm{Median} \mathrm{vier} \mathrm{neue} \mathrm{Läsionen} \mathrm{und} \mathrm{für} \mathrm{Typ} 4$ fünf neue Läsionen. Die Klassifikation der DSA zeigte für Typ 1 vier neue Läsionen im Median gegenüber drei neuen Läsionen für Typ 4. Übereinstimmend in CTA und DSA sind also die Typen 1 
und 4 nach Lovett et al. im Median mit den meisten Läsionen nach SPAC vergesellschaftet.

Tabelle 10: Kreuztabelle der Ulzerationen in der DSA und in der CTA. Berechnet sind daraus Sensitivität und Spezifität.

\begin{tabular}{|c|c|c|c|c|}
\hline \multirow{2}{*}{\multicolumn{2}{|c|}{ Ulzeration Konsens }} & \multicolumn{2}{|c|}{ Ulzeration DSA } & \multirow[b]{2}{*}{ Gesamt } \\
\hline & & - & + & \\
\hline \multirow[t]{2}{*}{ Ulzeration CTA } & - & 16 & 1 & 17 \\
\hline & + & 6 & 18 & 24 \\
\hline Gesamt & & 22 & 19 & 41 \\
\hline
\end{tabular}

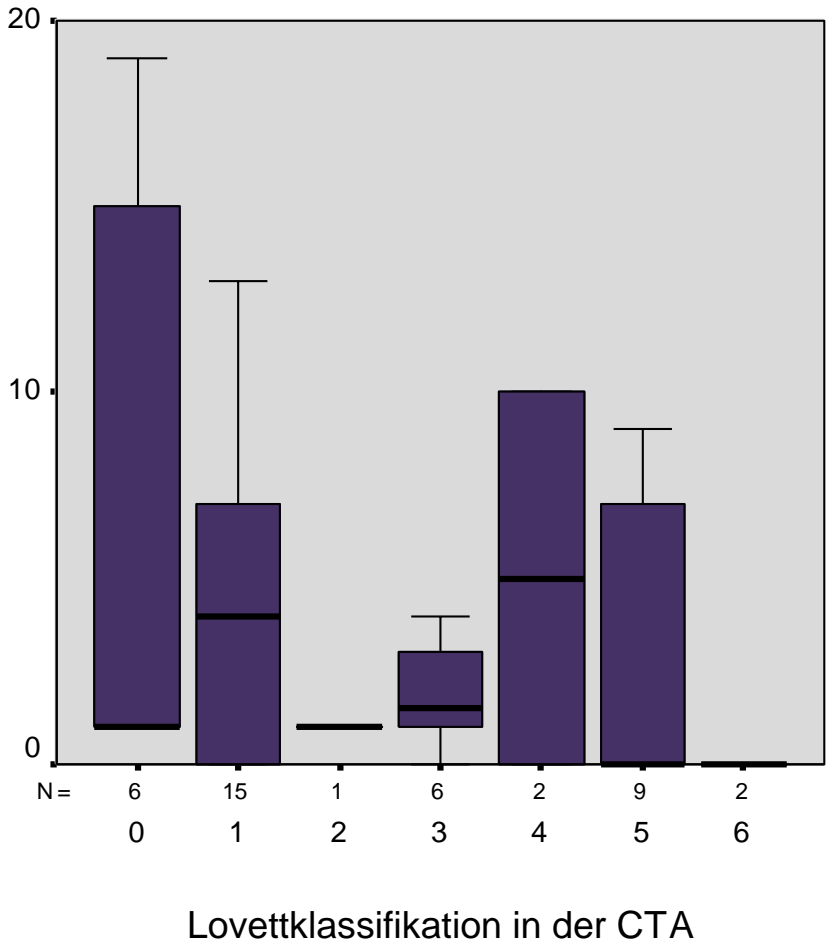

Abbildung 13: Boxplot. Dargestellt ist die Anzahl neuer DWI-Läsionen für die einzelnen Typen der Klassifikation nach Lovett et al. in der CT-Angiographie. Die Typen 1 und 4 haben dabei im Median die meisten Läsionen. 


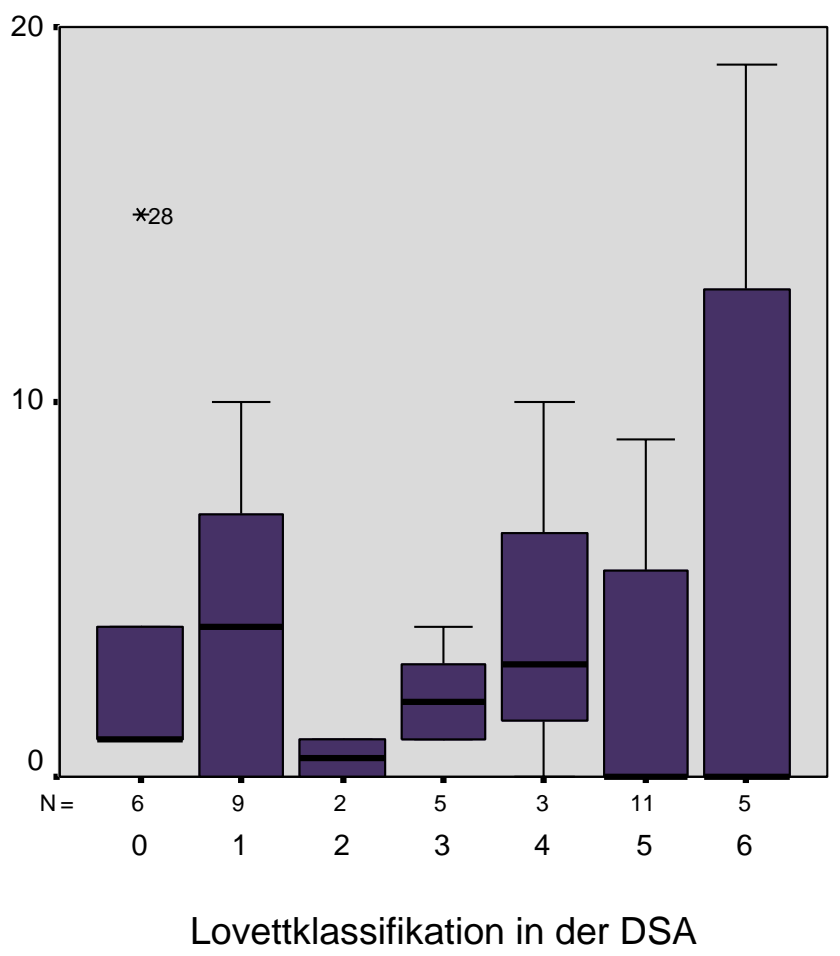

Abbildung 14: Boxplot. Dargestellt ist die Anzahl neuer DWI-Läsionen für die einzelnen Typen der Klassifikation nach Lovett et al. in der Digitalen Subtraktionsangiographie. Die Typen 1, 3 und 4 gehen dabei im Median mit den meisten Läsionen einher.

\subsection{Einsatz von zerebralen Protektionssystemen}

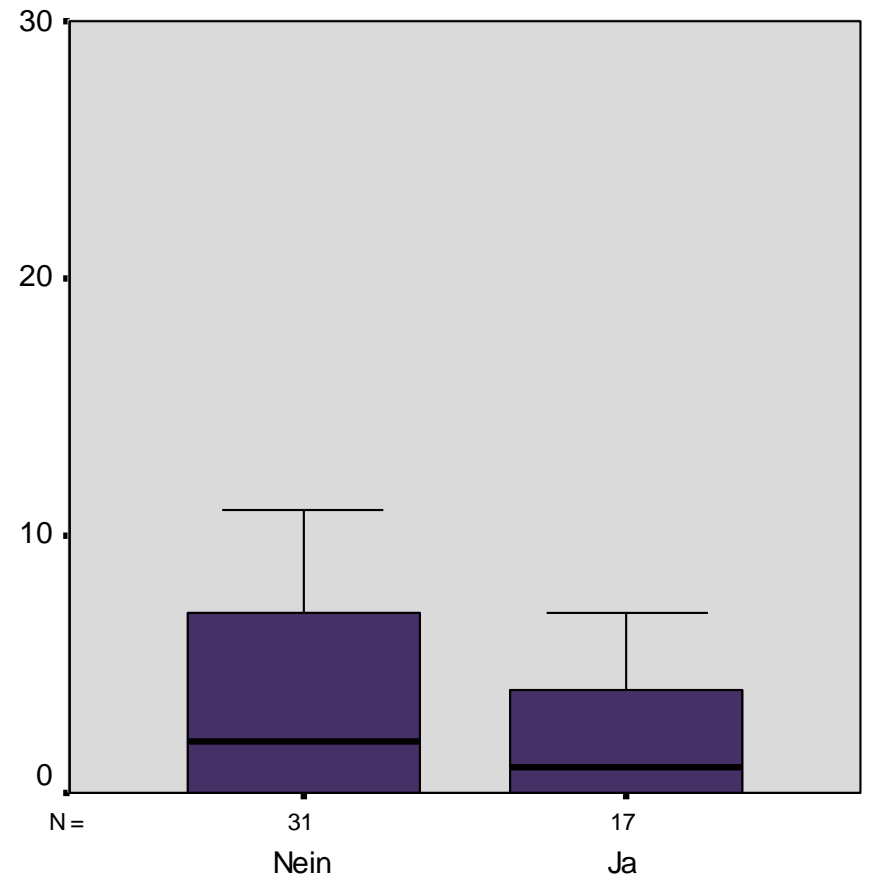

Einsatz eines Protektionssystems

Abbildung 15: Boxplot. Dargestellt sind Anzahl neuer DWI-Läsionen nach Intervention für Patienten, die eine SPAC mit zerebraler Protektion zugeführt wurden und Patienten ohne zerebrale Protektion. 
In 17 Fällen (35,42\%) wurde ein Protektionssystem verwendet. In 12 dieser Fälle traten DWI-Läsionen auf. Protektionssysteme konnten somit die Häufigkeit von DWILäsionen nach SPAC nicht senken (s. Tabelle 12). Die Anzahl neuer Läsionen lag in der Gruppe mit Protektionssystemen bei 1 gegenüber 2 in der Gruppe ohne Protektion (s. Abbildung 15). Damit konnte die Anzahl neuer Läsionen leicht gesenkt werden.

\subsection{Technische Details der SPAC}

Die Dauer der Angioplastie und Stentimplantation lag im Durchschnitt bei 28,55 min (min. 15 min; max. 69 min) und es wurden im Mittel 6 Kontrastmittel-Serien (min. 4; max. 14) angefertigt. In der Tabelle 11 sind diese Risikofaktoren der SPAC jeweils für die Gruppen der Patienten mit und ohne neue DWI-Läsionen widergegeben. Es zeigte sich, dass die Interventionen bei Patienten mit neuen Läsionen nach SPAC im Mittel länger waren (29,07 min gegenüber 27,65 min, p-Wert= 0,199) und dass auch die Anzahl der Kontrastmittelserien signifikant größer war. In der Gruppe der Patienten mit neuen Läsionen wurden im Mittel 6,74 Kontrastmittelserien angefertigt und in der Gruppe ohne Läsionen dagegen 5,59 ( $p-$ Wert= 0,036).

Tabelle 11: Risikofaktoren der SPAC für periinterventionelle Mikroembolien.

\begin{tabular}{lcccc}
\hline \hline & $\begin{array}{l}\text { Keine neuen } \\
\text { DWI-Läsionen }\end{array}$ & $\begin{array}{l}\text { Neue DWI- } \\
\text { Läsionen }\end{array}$ & Odds Ratio & p-Wert \\
\hline $\begin{array}{l}\text { Mittlere Länge der } \\
\text { Intervention }\end{array}$ & 27,65 min & 29,07 min & - & 0,199 \\
$\begin{array}{l}\text { Mittlere Anzahl } \\
\text { von Serien }\end{array}$ & 5,59 & 6,74 & - & $0,036^{*}$ \\
$\begin{array}{l}\text { Entlüftung } \\
\text { Protektionssystem }\end{array}$ & $5(10,42 \%)$ & $12(25 \%)$ & 1,52 & 0,428 \\
Ulzeration & $5(10,42 \%)$ & $14(29,17 \%)$ & 1,94 & 0,519 \\
\hline \hline * auf dem Niveau von $0,05(2-s e i t g)$ signifikant. & & & 0,48 \\
\hline
\end{tabular}

* auf dem Niveau von 0,05 (2-seitig) signifikant.

Entlüftung des Stentsystems war zwar in beiden Gruppen gleich häufig vertreten, konnte die Anzahl von Mikroembolien aber senken ( $\mathrm{OR}=0,48)$. Die mittlere Anzahl neuer DWI-Läsionen war in der Gruppe mit Entlüftung signifikant niedriger (s. 
Abbildung 16) als in der Gruppe ohne Entlüftung (1,13 gegenüber 4,15; $p$-Wert= $0,012)$.

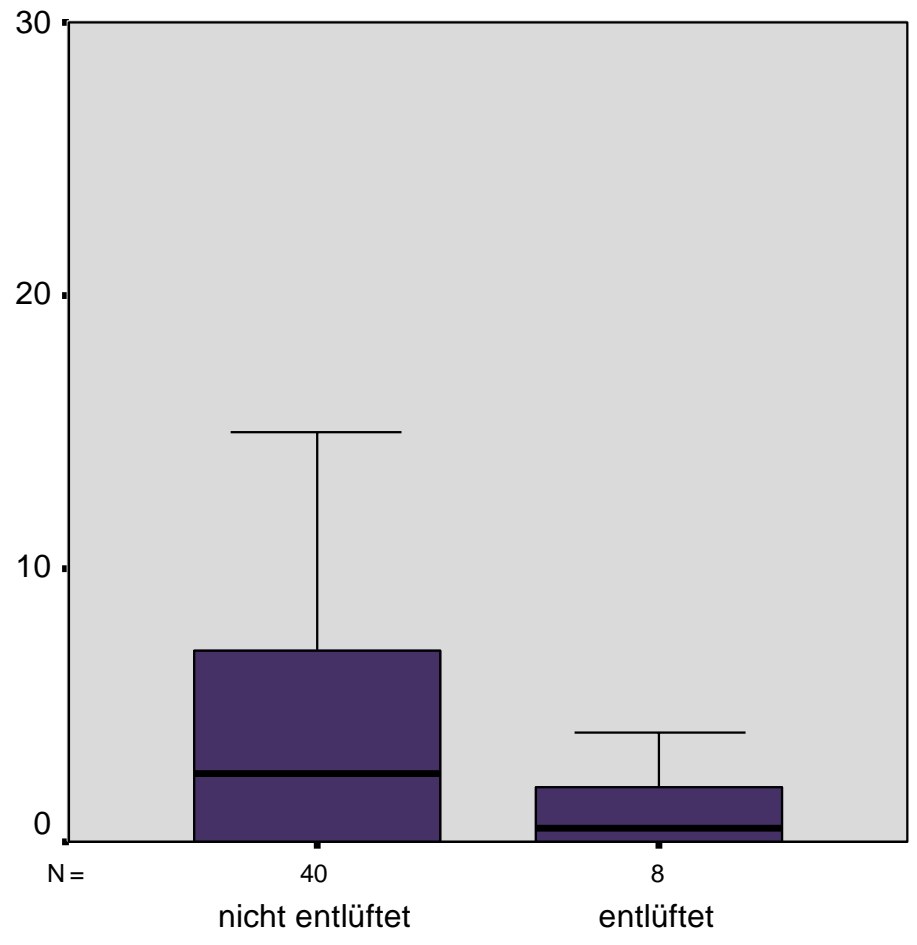

\section{Entlüftung}

Abbildung 16: Boxplot. Dargestellt ist die Anzahl neuentstandener DWI-Läsionen nach SPAC für Interventionen, in denen das Stentsystem vor Applikation entlüftet und in denen das System nicht entlüftet wurde. 


\section{$\underline{\text { 6. Diskussion }}$}

Ziel unserer prospektiven Studie war, prädiktive Faktoren für das Auftreten von mikroembolischen Ereignissen während der SPAC zu gewinnen. Parameter zum Stenosegrad und zur Plaquemorphologie wurden dafür aus CT-Angiographie und Digitaler Subtraktionsangiographie gewonnen. Wir haben 46 Patienten einer SPAC zugeführt, von denen 2 Patienten an beiden Karotiden behandelt wurden. Vor und nach SPAC angefertigte zerebrale DWI-MRT wurden hierzu verglichen, um periinterventionelle Mikroembolien zu ermitteln.

\subsection{SPAC}

In allen 48 Fällen wurde die stenosierte Arterie durch einen Stent wieder rekanalisiert. Da in 3 Fällen nach Intervention eine klinische Komplikation auftrat (1 TIA, 1 minor stroke und 1 major stroke), liegt unsere Komplikationsrate bezüglich klinisch manifester neurologischer Defizite bei 6,25\%. Für symptomatische Karotisstenosen liegt sie bei 5,89\% und für asymptomatische bei 7,14\%. Damit liegt die Komplikationsrate in unserem Zentrum unter der Komplikationsrate der Studien des Global Carotid Stent Registry $(7,8 \%)$ und des Pro-CAS (Prospektive Registry of Carotid Angioplasty and Stenting) (8,8\%) (Wholey et al. 2003, Theiss et al. 2004) und ist mit der Komplikationsrate der TEA vergleichbar (7,1\% für symptomatische und 2,9\% für asymptomatische Karotisstenosen aus Rothwell et al. 2003 und Wholey et al. 2005). DWI-Läsionen konnten bei 31 Patienten (64,6\%) beobachtet werden. Dies ist weit höher als in anderen Studien, bei denen im Durchschnitt 37\% der Patienten neue Läsionen aufwiesen (Schnaudigel et al. 2008). Jüngste Studien, darunter Krapf et al. (2006) und Tedesco et al. (2007), berichteten allerdings mit 56,8\% bzw. $70 \%$ über ähnlich hohe Raten. Interessanterweise traten in unserer Studie in 14 Fällen $(29,17 \%)$ DWI-Läsionen außerhalb des Versorgungsgebietes der behandelten Arterie auf. Andere Studien beobachteten dieses Phänomen ebenfalls (Schnaudigel et al. 2008). Dies deutet daraufhin, dass nicht nur die Plaques der behandelten Stenose, sondern auch Plaques im Bereich des Aortenbogens oder Luftembolien durch den Katheter der Ursprung von Mikroembolien sind. Tedesco et al. berichteten über ein erhöhtes signifikantes Auftreten von DWI-Läsionen nach Darstellung des 
Aortenbogens in der Angiographie und Gröschel et al. (2008) machten Kalzifikationen der Aorta als prädiktive Faktoren für Läsionen nach SPAC aus.

Zum ersten Mal wurden auch technische Details der SPAC wie Anzahl der angefertigten Kontrastmittelserien, Länge der Intervention und Entlüftung des Stentsystems hinsichtlich ihres Einflusses auf periinterventionelle Mikroembolien untersucht. Es zeigte sich, dass in der Gruppe der Patienten, die neue Läsionen nach SPAC hatten, signifikant mehr Kontrastmittelserien angefertigt wurden als in der Gruppe ohne Läsionen (5,59 gegenüber 6,74; p-Wert=0,036, s. Tabelle 12). Ebenso dauerte die Intervention etwas länger (27,65 min gegenüber 29,07 min). Daraus folgern wir, dass Länge der Intervention und Anzahl der Kontrastmittelserien Risikofaktoren oder Indikatoren von Risikofaktoren für mikroembolische Ereignisse sind. Dies und die Tatsache, dass auch außerhalb des Gefäßterritoriums Läsionen entstehen, zeigen außerdem, dass die Plaquemorphologie der Karotisstenose nicht als alleiniges prädiktives Maß für das Risiko von mikroembolischen Ereignissen nach SPAC gelten kann. Unklar ist aber, ob es sich bei diesen Mikroembolien um eine Abschwemmung von thrombotischem Material aus dem Aortenbogen handelt oder um Luftembolien durch den Katheter und das Stentsystem. Um die Bedeutung von Luftembolien in unserer Studie zu untersuchen, verglichen wir Patienten bei denen das Stentsystem entlüftet wurde mit Patienten, bei denen es nicht entlüftet wurde. Es zeigte sich, dass unter Entlüftung signifikant weniger DWI-Läsionen nach SPAC auftraten. So lag der Mittelwert in der Gruppe mit Entlüftung bei 1,13 während er in der Gruppe ohne Entlüftung bei 4,15 lag ( $p-W e r t=0,012)$. Dies deutet daraufhin, dass Luftembolien einen nicht unbeträchtlichen Anteil an DWI-Läsionen nach SPAC haben.

\subsection{Zerebrale Protektionssysteme}

Wir untersuchten in unserer Studie den Einfluss von zerebralen Protektionssystemen auf das Auftreten von Mikroembolien nach SPAC. Dazu führten wir 17 Patienten einer SPAC mit Protektion zu. Bislang lässt die Studienlage keine eindeutige Empfehlung für Protektionssysteme zu. Da Mikroembolien nicht nur während des Passierens des Stentsystems oder des Freisetzens des Stents entstehen, kann ein zerebrales Protektionssystem keinen sicheren Schutz vor diesen darstellen. Vor allem nicht, wenn es sich um Luftembolien handelt. Die Frage nach der Bedeutung 
eines zerebralen Protektionssystems ist noch nicht abschließend geklärt. Kastrup et al. (2003) berichteten in einer Metaanalyse, dass die 30-Tage Inzidenz von Schlaganfall und Tod in der Gruppe von SPAC mit Protektionssystem niedriger $(1,8 \%)$ gegenüber der Gruppe von SPAC ohne Protektion war. Allerdings spielen hier auch Lernkurveneffekte eine Rolle. Wholey et al. (2003) berichteten ein ähnliches Ergebnis. Die SAPHIRE-Studie (Stenting and Angioplasty in Patients at High Risk for Endarterectomy) konnte sogar zeigen, dass die Rate von Schlaganfällen in der Gruppe der SPAC mit zerebraler Protektion und der Gruppe der TEA keine Unterschiede zeigt. Bezüglich der Inzidenz von Mikroembolien berichteten Kastrup et al. (2003), dass Protektionssysteme die Inzidenz neuer DWI-Läsionen senken konnten (67\% in der Gruppe der SPAC ohne Protektion vs. $49 \%$ mit Protektion). Da in unserer Studie 12 der 17 Patienten, die einer SPAC mit zerebraler Protektion zugeführt wurden, mindestens eine neue Läsion in der DWI-MRT aufwiesen, können wir die genannten Ergebnisse nicht vorbehaltlos unterstreichen. Interessanterweise zeigte die Gruppe mit Protektionssystem allerdings im Median weniger neue DWILäsionen als die ohne Protektion (1 vs. 2). Wir schließen daraus also auch, dass Protektionssysteme einen Einfluss auf das Auftreten von Mikroembolien nach SPAC haben. Der Einsatz von Protektionssystemen hat also zwar keinen Einfluss auf die Inzidenz mind. 1 neuen mikroembolischen Ereignisses nach SPAC, jedoch können Protektionssysteme möglicherweise die Anzahl dieser Läsionen senken.

$\mathrm{Da}$ jedoch die mikroembolischen Ereignisse in fast allen Fällen keine klinisch erkennbaren Symptome verursachen, ist der klinische Nutzen der Protektionssysteme in der Verhinderung oder der Reduktion der Häufigkeit der sehr viel selteneren makroembolischen Komplikationen.

Jansen 0 et al. (2009) analysierten Daten aus der SPACE-Studie (stent-protected angioplasty versus carotid endarterectomy in symptomatic patients), in der 418 Patienten einer SPAC mit Protektion und 145 Patienten einer SPAC ohne Protektion zugeführt wurden. Es wurden keine Unterschiede zwischen beiden Gruppen bezüglich der Prävention eines ipsilateralen Schlaganfalls innerhalb der ersten 30 Tage festgestellt. Einen kleinen Benefit durch Protektionssysteme ergab sich lediglich in einer Subgruppe von Patienten, die einer SPAC mit einem sogenannten „open cell stent“ mit einer Porengröße $>2,5 \mathrm{~mm}^{2}$ zugeführt wurden. 


\subsection{CTA und DWI-MRT}

Das von uns entwickelte Verfahren zur Bestimmung des Weichplaqueanteils der Plaque in der CTA hat sich im Laufe der Studie gut bewährt. Es erlaubt nicht nur die Bestimmung des Weichplaqueanteils, sondern auch die Bestimmung der Anzahl der Hartplaques bzw. Hartplaquespangen, der Volumina von Hart-, Weich- und Gesamtplaque, der Länge der Stenose, sowie die Erkennung von Ulzerationen. Ungenauigkeiten ergeben sich vor allem durch die unterschiedliche Qualität der Untersuchung. Dies kann auf Artefakten durch Zahnimplantate beruhen, aber auch auf unterschiedliches Anfluten des Kontrastmittels. Letzteres ist zwar perfusorgesteuert, trotzdem ergeben sich Unterschiede in der HE des kontrastmittelangereicherten Blutes durch unterschiedliche Auswurffraktionen des Herzens. Wichtig bei dieser Methode ist, dass der Untersucher ein für sich optimales Fenster einstellt. Dabei muss er sich zwar an das Protokoll halten, es wird inm aber dafür ein gewisser Spielraum gewährt.

Es ist nach der momentanen Studienlage noch nicht einheitlich geklärt, welche HE eine Hartplaque definiert. Einige Studien gehen schon von einer HE von 110 aus, Nandalur et al. (2005) von 130, Saba et al. (2007) von 120 und Silvennoinen et al. (2007) von 200-300 HE. Nandalur et al. berichteten allerdings, dass eine Plaque erst ab einer Dichte von $350 \mathrm{HE}$ gut als solche erkannt werden kann. Wir definierten eine Hartplaque daher ab einer HE von 300. In der Quantität des Hartplaqueanteils kann es damit in der Vergleichbarkeit der Studien untereinander Schwierigkeiten geben.

Die Bestimmung von DWI-Läsionen im MRT ist ein recht einfaches Verfahren, das sich in dieser Form schon in vielen Studien bewährt hat (Krapf et al. 2006, Zahn et al. 2007). Schwäche dieses Verfahrens ist, dass es nur die Fläche jeder einzelnen Läsion bestimmen kann, nicht jedoch das Volumen. Kleine Läsionen könnten je nach Schichtdicke zwischen den Schichten verloren gehen. Ebenso ist darauf zu achten, dass die Schichten vor und nach der Intervention genau in der Lokalisation übereinstimmen.

In hausinternen Studien hat sich außerdem gezeigt, dass auch die Feldstärke des Magnetfeldes (1.5- versus 3.0-T-Hochfeld-MRT) einen Einfluss auf das Erkennen mikroembolischer Ereignisse in der DWI-MRT hat. So hat die Diffusionsgewichtung im 3.0-T-MRT ein höheres Signal-zu-Rausch-Verhältnis und eine höhere 
Ortsauflösung. Es konnte gezeigt werden, dass im 3.0-T-MRT mehr DWI-Läsionen nachweisbar sind als in zeitgleich durchgeführten 1.5-T-MRT- Bildern.

\subsection{Zusammenhang Plaquemorphologie und DWI-Läsionen}

Bisher unklar waren der Einfluss der Plaquezusammensetzung und die damit verbundene Instabilität auf Komplikationen während der SPAC. In unserer Studie fanden wir Anhaltspunkte dafür, dass der Weichplaqueanteil einen Einfluss auf das Auftreten von mikroembolischen Ereignissen hat. Zum einen hatten Patienten mit hohem Weichplaqueanteil mehr Läsionen in der DWI-MRT nach Intervention als Patienten mit niedrigem Weichplaqueanteil (s. Tabelle 08). Diese Korrelation ist in Abbildung 07 deutlich zu sehen, erreicht allerdings bei niedriger Fallzahl nicht das Signifikanzniveau von $5 \%(p=0,094)$. Wir erwarten, dass eine Studie mit einer höheren Fallzahl hierfür ein deutlicheres und signifikantes Ergebnis liefern würde. Zum anderen beeinflusst ein hoher Weichplaqueanteil die Gesamtfläche der DWILäsionen nach Intervention (s. Tabelle 09). In unserer Studie korrelierte der Weichplaqueanteil positiv signifikant mit der Gesamtfläche der DWI-Läsionen nach Intervention $(p-W e r t=0,045)$. Außerdem hatten Patienten, die DWI-Läsionen aufwiesen, im Mittel mit 77,53\% gegenüber 65,9\% einen höheren Weichplaqueanteil als Patienten, die keine neuen Läsionen hatten (s. Abbildung 09). Der Weichplaqueanteil hat also möglicherweise einen prädiktiven Wert sowohl bezüglich der Anzahl und der Gesamtfläche von Mikroinfarkten nach SPAC als auch bezüglich der Wahrscheinlichkeit, ein mikroembolisches Ereignis nach SPAC zu erleiden.

Die Anzahl der Hartplaques korrelierte in unserer Studie negativ mit der Anzahl und Fläche neuer Läsionen. Mit einem p-Wert von 0,164 bzw. 0,084 ist dieses Ergebnis zwar nicht signifikant und sicher auch dadurch beeinflusst, dass ein erhöhter Hartplaqueanteil mit der Anzahl der einzelnen Hartplaques korreliert. Trotzdem vermuten wir, dass die Zahl der Hartplaquespangen die Stabilität der Plaque während der SPAC positiv beeinflusst. Dass Kalzifikationen der Plaque einen Einfluss auf die Stabilität und die Klinik der Patienten hat, konnten zahlreiche Studien bereits zeigen (Nandalur et al. 2006). Hunt et al. (2002). beobachteten weniger Schlaganfälle und TIA bei Patienten mit kalzifizierten Plaques. Sie berichteten auch, dass kalzifizierte Plaques seltener ulzeriert waren. Ebenso Nandalur et al. (2005), die sogar 21 Mal weniger Symptome bei diesen Patienten sahen. Untersuchungen an 
Plaques in Koronargefäßen kamen zu ähnlichen Schlüssen. Wexler et al. (1996) folgerten, dass die Kalzifikationen der Plaques, diese gegen mechanischen und biochemischen Stress resistenter machen. Fuster et al. (2005b) vermuteten, dass Kalzifikationen der Plaque eher Ausdruck des Umbaus und der Regeneration von Läsionen innerhalb der Plaque sind, als dass sie eine vulnerable Hochrisikoplaque darstellen.

\subsection{Ulzerationen und Lovettklassifikation als Risikofaktoren}

In unserer Studie konnten wir zeigen, dass ein Zusammenhang zwischen ulzerierten Plaques und DWI-Läsionen nach SPAC besteht (s. Abbildung 10). Ulzerierte Plaques gingen dabei im Median mit mehr DWI-Läsionen nach SPAC einher als nicht ulzerierte Plaques (3 gegenüber 1 Läsion). Ebenso waren ulzerierte Plaques in der Gruppe der Patienten mit neuen DWI-Läsionen nach SPAC mit 14 (29,17\%) deutlich häufiger vertreten als in der Gruppe ohne Läsionen mit 5 (10,42\%) (s. Tabelle 11). Daraus ergab sich eine Odds-Ratio von 1,94, die allerdings nicht signifikant war. Diese Zahl und die Tatsache, dass Plaqueulzerationen einen Einfluss auf kardio- und zerebrovaskuläre Ereignisse haben, lassen vermuten, dass Plaqueulzerationen auch einen Einfluss auf das Auftreten von Mikroembolien während SPAC haben und damit ein möglicher Risikofaktor hierfür sind. Eine Studie mit einer höheren Fallzahl würde womöglich auch hierfür ein deutlicheres und signifikantes Ergebnis liefern.

Dass die Plaquestabilität und damit verbundene Ulzerationen einen wesentlichen Einfluss auf das Auftreten von TIA und Schlaganfällen hat, konnte in vielen Studien bereits gezeigt werden (Ballotta et al. 2000, Avril et al. 1991, Sterpetti et al. 1991, Park et al. 1998). Die angiographische Erscheinung von Ulzerationen konnte auch in Koronargefäßen eine prädiktive Aussage über ihre Stabilität machen (Rapp et al. 2007, Jansen C et al. 1994, Cantelmo et al. 1998, Barth et al. 2000). Pathogenetisch vermutet man, dass die ulzerierte Plaqueoberfläche die Basis von Thromben ist und so zu Embolien führt oder durch Ausschüttung von thrombogenen Substanzen zu lokalen Thrombosen führt. Möglich ist allerdings auch, dass eine Ulzeration der Plaque lediglich ein Indikator für ihre Stabilität darstellt.

Krapf et al. (2006) berichteten allerdings, dass die Plaqueoberfläche, durch Ultraschall untersucht, keinen Einfluss auf zerebrale Schäden nach SPAC hat. Dies könnte auch auf Unterschieden in der Darstellung von Ulzerationen in der CTA und in 
der Sonographie beruhen. In der CTA lassen sich gerade auf den axialen Schichten selbst kleine Unregelmäßigkeiten der Gefäßwand sehr gut darstellen. Saba et al. (2007) berichteten eine Sensitivität der CTA von 93,9\% bei einer Spezifität von $98,7 \%$ in der Darstellung von Ulzerationen. Dabei handelte es sich um Präparate nach TEA, die histopathologisch untersucht wurden. Schwierigkeiten bei der Bestimmung von Ulzerationen in der Plaque ergeben sich allerdings bei stark kalzifizierten Plaques bzw. bei Ulzerationen, die unmittelbar an eine Hartplaque angrenzen; auch wenn diese selten sind. Auch das berichteten Saba et al., was wir in unseren Messungen bestätigen konnten.

Wir haben gezeigt, dass sich die Einteilung von Plaques nach Lovett et al. in der CTA sehr gut reproduzieren lässt. In den meisten Fällen (31 von 41) stimmte die Klassifikation der CTA mit der DSA überein. Insbesondere in Hinblick auf das Auffinden von Ulzerationen innerhalb der Plaques. Hier zeigte die CTA eine Sensitivität von 94,7\% und eine Spezifität von 72,7\% (s. Tabelle 10).

Dass die Klassifikation der CTA mit der DSA zwar in den meisten, aber nicht in allen Fällen übereinstimmt, ist vor allem auf die Ähnlichkeit der Typen 0, 5 und 6 zurückzuführen. Alle drei Typen beschreiben eine nicht ulzerierte Stenose, doch es ist nicht immer einfach zu bestimmen, ob der Gefäßverlauf bzw. die Gefäßwand glatt ist oder doch unregelmäßig. Zumal es auch fließende Übergänge zwischen diesen Typen gibt. Trotz definierter Kriterien ist die Einteilung in diesen Grenzfällen subjektiv.

Die prognostisch wichtigere Bestimmung der Ulzeration ist allerdings sehr gut zu reproduzieren. Zwar kann bei einem gebogenen Gefäßverlauf eine nach oben oder nach unten verlaufende Ulzeration mit einer geraden Ulzeration verwechselt werden, doch lassen sich die Ulzerationen sehr gut lokalisieren und als solche erkennen. Saba et al. beschrieben Schwierigkeiten beim Auffinden von Ulzerationen in stark kalzifizierten Bereichen. Auch wir können über solche Schwierigkeiten berichten.

Unser Vergleich der Lovetttypen untereinander bezüglich des Aufftretens neuer DWILäsionen lässt die Vermutung zu, dass die Morphologie der Ulzerationen einen Einfluss auf die Stabilität der Plaques haben könnte. Es zeigte sich, dass gerade die Typen 1 bis 4, also die Typen, die eine Ulzeration beschreiben, im Median mehr neue DWI-Läsionen aufwiesen (s. Abbildungen 13 und 14). Die Typen 1 und 4 hatten sogar die meisten Läsionen, doch sind unsere Patientenzahlen zu gering, um eine konkrete Aussage über die einzelnen Typen nach Lovett et al. als prädiktive Faktoren 
zu machen. Es konnte schon in einigen Studien ein Zusammenhang zwischen ulzerierten Plaques und Schwere der Klinik bei Karotisstenosen nachgewiesen werden und damit auf die Plaquestabilität geschlossen werden. Wir konnten mit unserer Studie zeigen, dass dieser Zusammenhang insbesondere auch bei der Behandlung mit SPAC gilt.

\subsection{Andere Risikofaktoren}

Dass der Stenosegrad einen Einfluss auf das Auftreten von zerebralen Schäden nach SPAC hat, liegt nahe. Als Ursache dafür ist vorstellbar, dass das Risiko thrombotisches Material abzuschwemmen beim Passieren des Katheters oder des Protektionssystems durch die Enge der Stenose erhöht wird. Ebenso beim Dilatieren des Gefäßes und beim Freisetzen des Stents, da eine enge Stenose einem höheren mechanischem Stress ausgesetzt ist um sie zu rekonstruieren. Wir konnten jedoch keinen statistischen Zusammenhang zwischen dem Grad der Enge und dem Auftreten von DWI-Läsionen nach SPAC erkennen. Krapf et al. (2006) sahen in ihrer Studie ebenso keinen Zusammenhang, genau wie du Mesnil de Rochemont et al. (2006). Krapf et al. (2006) beschrieben sogar eine viel niedrigere Chance für 3 oder mehr DWI-Läsionen bei subtotalen Stenosen.

Umstritten ist bislang, ob das Alter ein Risiko für mikroembolische Ereignisse bei SPAC darstellt; vor allem bei Patienten über 70 Jahren. Zahn et al. (2007) beobachteten eine höhere Komplikationsrate bei über 70-Jährigen. Wir konnten in unserer Studie bei Patienten über 70 Jahren ebenfalls ein höheres Risiko für mikroembolische Ereignisse beobachten $(\mathrm{OR}=2,05)$.

Ein höheres Risiko fanden wir außerdem für Männer $(O R=2,84)$ und für asymptomatische Stenosen ( $\mathrm{OR}=2,57)$, wobei dieses Ergebnis nicht signifikant ist und wir hierfür auch keinen kausalen Zusammenhang sehen. Obwohl Statine als Plaquestabilisatoren bei KHK und Karotisstenosen gelten, konnten wir keine Senkung des Risikos bei Statintherapie beobachten. Dazu ist allerdings anzumerken, dass uns keine Daten über den Beginn der Statintherapie unserer Patienten vorlagen.

Interessanterweise hatten Patienten, die an der rechten Karotis behandelt wurden, mehr Läsionen als die, die an der linken Karotis behandelt wurden $(\mathrm{OR}=3,47, \mathrm{p}$ Wert $=0,059)$. Bisher berichteten noch keine anderen Studien über diesen 
Unterschied. Eine Erklärung hierfür könnte die unterschiedliche Konfiguration im Abgang aus dem Aortenbogen zwischen rechter und linker A. carotis sein. Der höherlumige Abgang als Truncus brachiocephalicus mit dem steilen Abgang der A. carotis communis dextra könnte eine Passage von embolischem Material aus dem Aortenbogen begünstigen. 


\section{Zusammenfassung}

Unsere Studie ist die erste Studie, die den Zusammenhang zwischen Weichplaqueanteil der Plaques von symptomatischen und asymptomatischen Karotisstenosen und periinterventioneller mikroembolischer Ereignisse per DWI-MRT bei der SPAC untersuchte. Des Weiteren untersuchten wir den Zusammenhang zwischen Plaqueulzerationen und Mikroembolien nach SPAC. Dafür klassifizierten wir die Morphologie der Plaque nach der Klassifikation von Lovett et al.. Wir versuchten diese Klassifikation, die ursprünglich anhand der DSA entwickelt wurde, in der CTA zu reproduzieren. Aus unseren klinischen Patientendaten und Messdaten aus CTA und DSA untersuchten wir weitere Risikofaktoren für das Auftreten von Mikroembolien nach SPAC. Wir untersuchten hierfür nicht nur ihren Einfluss auf die Inzidenz dieser Ereignisse nach SPAC, sondern auch auf die Quantität in Größe und Anzahl der Läsionen. Des Weiteren untersuchten wir Details der technischen Durchführung der SPAC wie Entlüftung, Kontrastmittelserien oder den umstrittenen Einsatz von zerebralen Protektionssystemen.

Wir fanden wesentliche Hinweise, dass der Weichplaqueanteil der Karotisplaques einen Einfluss auf das Auftreten von mikroembolischen Ereignissen nach SPAC hat. Je größer der Weichplaqueanteil, desto höher ist die Inzidenz von DWI-Läsionen nach SPAC. Außerdem erhöhten sich mit dem Weichplaqueanteil auch die Anzahl der DWI-Läsionen und vor allem deren Gesamtfläche. Damit sagt der Weichplaqueanteil auch, in gewisser Hinsicht, etwas über die mögliche Schwere der mikroembolischen Ereignisse aus. Die Anzahl von Hartplaques scheint ebenfalls eine Aussage über die Prognose der SPAC zu machen. So sank mit der Anzahl an Hartplaques die Zahl neuer DWI-Läsionen nach SPAC. Wir vermuten, dass Hartplaques bzw. Hartplaquespangen wesentlich zur Stabilität der Plaques während der Intervention beitragen.

Einen weiteren Einfluss auf das Auftreten von DWI-Läsionen nach SPAC hatte die Plaquemorphologie hinsichtlich Ulzerationen der Plaques. Wir konnten zeigen, dass Ulzerationen die Wahrscheinlichkeit des Auftretens mindestens einer Läsion nach SPAC erhöht und auch deren Anzahl bei betroffenen Patienten. Damit konnten wir die Ergebnisse anderer Studien über die Bedeutung der Ulzeration der Plaque für mikroembolische Ereignisse unterstreichen. Des Weiteren konnten wir zeigen, dass sich die Plaquemorphologie in der CTA sehr gut beurteilen und dass sich die 
Klassifikation der Morphologie in der DSA nach Lovett et al. auch in der CTA sehr gut reproduzieren lässt. Dies ist ein weiteres Argument in Richtung Gleichwertigkeit der CTA gegenüber der DSA in der Diagnostik der Karotisstenose. Wir konnten zwar eine erhöhte Häufung von mikroembolischen Ereignissen nach SPAC für die Typen 1 und 4 nach Lovett beobachten und damit Vermutungen über die Gefährlichkeit dieser Typen äußern, doch sind unsere Patientenzahl und vor allem die Anzahl der Patienten in einzelnen Typen zu gering, um konkrete Aussagen dazu machen zu können.

Naheliegend und schon in vielen Studien untersucht, ist der Zusammenhang der technischen Durchführung der SPAC und mikroembolischen Ereignissen nach SPAC. Entlüftung des Stentsystems, Anzahl der Kontrastmittelserien, Länge der Intervention und Einsatz von Protektionssystemen haben einen Einfluss auf Mikroembolien nach SPAC. Die Dauer der Intervention ist dabei sicherlich von ihrer Schwierigkeit abhängig. Dabei ist eine kurze und schnelle Intervention, ebenso wie eine geringe Anzahl von Kontrastmittelserien mit einer Reduktion von mikroembolischen Ereignissen assoziiert. Entlüftung des Stentsystems kann zusätzlich die Anzahl neuer Läsionen reduzieren. Bezüglich des Einflusses von Protektionssystemen auf mikroembolische Ereignisse sind unsere Ergebnisse kontroverser. Sie konnten zwar in unserer Studie die Anzahl der DWI-Läsionen nach SPAC senken, doch zeigten über $70 \%$ der Patienten mit zerebraler Protektion mindestens eine neue Läsion in der DWI-MRT und damit mehr als in der Gruppe ohne zerebraler Protektion. So können wir abschließend die essentielle Wichtigkeit der zerebralen Protektion nicht bekräftigen, obwohl wir weiterhin auf die Möglichkeit der zerebralen Protektion nicht verzichten wollen.

Die SPAC wird weiterhin für Patienten mit bestimmten Kriterien eine wichtige Therapieoption in der Behandlung der Karotisstenose darstellen und möglicherweise bei einigen Patienten der operativen Therapie überlegen sein. So gilt es die individuellen Risikofaktoren für den Patienten sowohl der SPAC als auch der TEA genau zu kennen, um dem Patienten eine optimale Therapie mit Reduzierung der Komplikationen zukommen zu lassen. 


\section{Literaturverzeichnis}

Alberts M (2001): Results of a multicenter prospective randomized trial of carotid artery stenting versus carotid endarterectomy. Stroke $\underline{32}, 325$

Alvarez-Linera J, Benito-Leon J, Escribano J, Campollo J, Gesto R (2003): Prospective evaluation of carotid artery stenosis: elliptic centric contrast enhanced MR angiography and spiral CT angiography compared with digital subtraction angiography. Am J Neuroradiol $\underline{24}, 1012-9$

Asakura F, Kawaguchi K, Sakaida H, Toma N, Matsushima S, Kuraishi K, Tanemura H, Miura Y, Maeda M, Taki W (2006): Diffusion-weighted MR imaging in carotid angioplasty and stenting with protection by the reversed carotid arterial flow. AJNR Am J Neuroradiol 27 (4), 753-8

Avril G, Batt M, Guidoin R, Marois M, Hassen-Khodja R, Daune B, Gagliardi J M, Le Bas $P$ (1991): Carotid endarterectomy plaques: correlations of clinical and anatomic findings. Ann Vasc Surg $\underline{5}(1), 50-4$

Ballotta E, Da Giau G, Renon L (2000): Carotid plaque gross morphology and clinical presentation: a prospective study of 457 carotid artery specimens. J Surg Res $\underline{89}(1), 78-84$

Bammer R (2003): Basic principles of diffusion-weighted imaging. Eur J Radiol $\underline{45}(3)$, 169-84

Barnett H J, Taylor D W, Eliasziw M, Fox A J, Ferguson G G, Haynes R B, Rankin R N, Clagett G P, Hachinski V C, Sackett D L et al. (1998): Benefit of carotid endarterectomy in patients with symptomatic moderate or severe stenosis. North American Symptomatic Carotid Endarterectomy Trial Collaborators. N Engl J Med $\underline{339}(20), 1415-25$

Barth A, Remonda L, Lovblad K O, Schroth G, Seiler R W (2000): Silent cerebral ischemia detected by diffusion-weighted MRI after carotid endarterectomy. Stroke 31(8), 1824-8 
Bates E R, Babb J D, Casey D E, Jr., Cates C U, Duckwiler G R, Feldman T E, Gray W A, Ouriel K, Peterson E D, Rosenfield K et al.(2007): ACCF/SCAI/SVMB/SIR/ASITN 2007 clinical expert consensus document on carotid stenting: a report of the American College of Cardiology Foundation Task Force on Clinical Expert Consensus Documents (ACCF/SCAI/SVMB/SIR/ASITN Clinical Expert Consensus Document Committee on Carotid Stenting). J Am Coll Cardiol $\underline{49}(1), 126-70$

Bendszus M, Stoll G (2006): Silent cerebral ischaemia: hidden fingerprints of invasive medical procedures. Lancet Neurol $\underline{5}(4), 364-72$

Brooks W H, McClure R R, Jones M R, Coleman T, Breathitt L (2001): Carotid angioplasty and stenting versus carotid endarterectomy: randomized trial in a community hospital. J Am Coll Cardiol $\underline{38}(6), 1589-95$

Brooks W H, McClure R R, Jones M R, Coleman T, Breathitt L (2004): Carotid angioplasty and stenting versus carotid endarterectomy for treatment of asymptomatic carotid stenosis: a randomized trial in a community hospital.

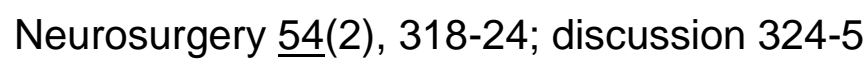

Buskens E, Nederkoorn P J, Buijs-Van Der Woude T, Mali W P, Kappelle L J, Eikelboom B C, Van Der Graaf Y, Hunink M G (2004): Imaging of carotid arteries in symptomatic patients: cost-effectiveness of diagnostic strategies. Radiology 233(1), $101-12$

Cantelmo N L, Babikian V L, Samaraweera R N, Gordon J K, Pochay V E, Winter M $R$ (1998): Cerebral microembolism and ischemic changes associated with carotid endarterectomy. J Vasc Surg 27(6), 1024-30; discussion 1030-1

Cercignani M, Horsfield M A (2001): The physical basis of diffusion-weighted MRI. J Neurol Sci 186 Suppl 1, 11-4

Chen Y, Manness W, Kattner K (2004): Application of CT Angiography of Complex Cerebrovascular Lesions during Surgical Decision Making. Skull Base 14, 185-93

Connors J J, Sacks D, Furlan A J, Selman W R, Russell E J, Stieg P E, Hadley M N, Wojak J C, Koroshetz W J, Heros R C, et al. (2005): Training, competency, and 
credentialing standards for diagnostic cervicocerebral angiography, carotid stenting, and cerebrovascular intervention: a joint statement from the American Academy of Neurology, the American Association of Neurological Surgeons, the American Society of Interventional and Therapeutic Neuroradiology, the American Society of Neuroradiology, the Congress of Neurological Surgeons, the AANS/CNS Cerebrovascular Section, and the Society of Interventional Radiology. Neurology 64(2), 190-8

Daugherty A, Rateri D L (2002): T lymphocytes in atherosclerosis: the yin-yang of Th1 and Th2 influence on lesion formation. Circ Res $\underline{90}(10), 1039-40$

Davies M J, Thomas A C (1985): Plaque fissuring--the cause of acute myocardial infarction, sudden ischaemic death, and crescendo angina. Br Heart J $\underline{53}$ (4), 363-73

Ding S, Zhang M, Zhao Y, Chen W, Yao G, Zhang C, Zhang P, Zhang Y (2008): The role of carotid plaque vulnerability and inflammation in the pathogenesis of acute ischemic stroke. Am J Med Sci $\underline{336}(1), 27-31$

du Mesnil de Rochemont R, Schneider S, Yan B, Lehr A, Sitzer M, Berkefeld J (2006): Diffusion-weighted MR imaging lesions after filter-protected stenting of highgrade symptomatic carotid artery stenoses. AJNR Am J Neuroradiol 27 (6), 1321-5

Eastcott H H (1954): Reconstruction of internal carotid artery in patient with intermittent attacks of hemiplegia. Lancet 1954, 2, 994-996

ECST = European Carotid Surgery Trialists' Collaborative Group (1991): MRC European Carotid Surgery Trial: interim results for symptomatic patients with severe (70-99\%) or with mild (0-29\%) carotid stenosis. Lancet 337(8752), 1235-43

ECST = European Carotid Surgery Trialists' Collaborative Group (1998): Randomised trial of endarterectomy for recently symptomatic carotid stenosis: final results of the MRC European Carotid Surgery Trial (ECST). Lancet 351(9113), 137987

Fayed A M, White C J, Ramee S R, Jenkins J S, Collins T J (2002): Carotid and cerebral angiography performed by cardiologists: cerebrovascular complications. Catheter Cardiovasc Interv $\underline{55}(3), 277-80$ 
Fiebach J B, Schellinger P D (2003): Moderne Kernspintechniken beim Schlangsanfall. Radiologe $\underline{43}, 251-264$

Fiebach J B, Schellinger P D, Jansen O (2002): CT and diffusion-weighted MR imaging (DWI) in randomized order: DWI results in higher accuracy and lower interter variability in the diagnosis of hyperacute ischemic stroke. Stroke $\underline{33}, 2206-10$

Fuster V, Moreno P R, Fayad Z A, Corti R, Badimon J J (2005a): Atherothrombosis and high-risk plaque: part I: evolving concepts. J Am Coll Cardiol 46 6 (6), 937-54

Fuster V, Fayad Z A, Moreno P R, Poon M, Corti R, Badimon J J (2005b): Atherothrombosis and high-risk plaque: Part II: approaches by noninvasive computed tomographic/magnetic resonance imaging. J Am Coll Cardiol 46 (7), 1209-18

Gauvrit J Y, Leclerc X, Girot M, Cordonnier C, Sotoares G, Henon H, Pertuzon B, Michelin E, Devos D, Pruvo J P, Leys D (2006): Fluid-attenuated inversion recovery (FLAIR) sequences for the assessment of acute stroke: inter observer and inter technique reproducibility. J Neurol $\underline{253}(5), 631-5$

Glor F P, Ariff B, Crowe L A, Hughes A D, Cheong P L, Thom S A, Verdonck P R, Firmin D N, Barratt D C, Xu X Y (2003): Carotid geometry reconstruction: a comparison between MRI and ultrasound. Med Phys $\underline{30}$ (12), 3251-61

Gröschel K, Pilgram S M, Ernemann U, Schnaudigel S, Nagele T, Knauth M, Kastrup A (2008): Aortic calcification on plain chest radiography predicts embolic complications during carotid artery stenting. Eur J Neurol $\underline{15}(7), 730-6$

Halliday A, Mansfield A, Marro J, Peto C, Peto R, Potter J, Thomas D (2004): Prevention of disabling and fatal strokes by successful carotid endarterectomy in patients without recent neurological symptoms: randomised controlled trial. Lancet 363(9420), 1491-502

Hunt J L, Fairman R, Mitchell M E, Carpenter J P, Golden M, Khalapyan T, Wolfe M, Neschis D, Milner R, Scoll B et al. (2002): Bone formation in carotid plaques: a clinicopathological study. Stroke $\underline{33}(5), 1214-9$

Jansen C, Ramos L M, van Heesewijk J P, Moll F L, van Gijn J, Ackerstaff R G (1994): Impact of microembolism and hemodynamic changes in the brain during 
carotid endarterectomy. Stroke 25(5), 992-7

Jansen O, Fiehler J, Hartmann M, Brückmann H (2009): Protection or nonprotection in carotid stent angioplasty: The influence of international Techniques on outcome data from the SPACE trial. Stroke $\underline{40}, 8411-6$

Johnston S C, Gress D R, Browner W S, Sidney S (2000): Short-term prognosis

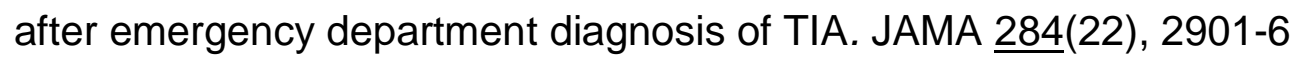

Kastrup A, Groschel K, Krapf H, Brehm B R, Dichgans J, Schulz J B (2003): Early outcome of carotid angioplasty and stenting with and without cerebral protection devices: a systematic review of the literature. Stroke $\underline{34}(3), 813-9$

Khalil M F, Wagner W D,Goldberg I J (2004): Molecular interactions leading to lipoprotein retention and the initiation of atherosclerosis. Arterioscler Thromb Vasc Biol 24(12), 2211-8

Krapf H, Nagele T, Kastrup A, Buhring U, Gronewaller E, Skalej M, Kuker W (2006): Risk factors for periprocedural complications in carotid artery stenting without filter protection: A serial diffusion-weighted MRI study. J Neurol 253(3), 364-71

Laubenberger TH, Laubenberger J: Technik der medizinischen Radiologie: Diagnostik, Strahlentherapie, Strahlenschutz: 7. Auflage; Deutscher Äzteverlag, Köln 1999, 355-371

Leonardi M, Cenni P, Simonetti L, Raffi L, Battaglia S (2005):. Retrospective study of complications arising during cerebral and spinal diagnostic angiography from 1998 to 2003. Intervent Neurol 11, 213-221

Levy E I, Mocco J, Samuelson R M, Ecker R D, Jahromi B S, Hopkins L N (2008):

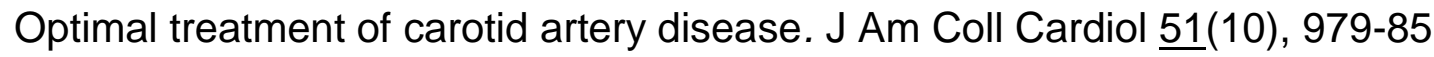

Libby P (2001): What have we learned about the biology of atherosclerosis? The role of inflammation. Am J Cardiol $\underline{88}(7 \mathrm{~B}), 3 \mathrm{~J}-6 \mathrm{~J}$

Lövblad K O, Pluschke W, Remonda L, Gruber-Wiest D, Do D D, Barth A, Kniemeyer H W, Bassetti C, Mattle H P, Schroth G (2000): Diffusion-weighted MRI

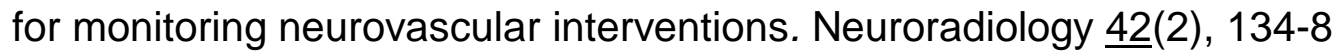


Lovett J K, Gallagher P J, Hands L J, Walton J, Rothwell P M (2004): Histological correlates of carotid plaque surface morphology on lumen contrast imaging. Circulation $\underline{110}(15), 2190-7$

Massachusetts Medical Society (1991): Beneficial effect of carotid endarterectomy in symptomatic patients with high-grade carotid stenosis. North American Symptomatic Carotid Endarterectomy Trial Collaborators. N Engl J Med $\underline{325}(7), 445-$ 53

Morgenstern L B, Fox A J, Sharpe B L, Eliasziw M, Barnett H J, Grotta J C (1997): The risks and benefits of carotid endarterectomy in patients with near occlusion of the carotid artery. North American Symptomatic Carotid Endarterectomy Trial (NASCET) Group. Neurology $\underline{48}(4), 911-5$

Mosso M, Baumgartner R (2000): Karotisstenose: Epidemiologie und Symptomatologie. Schweiz Med Wochenschr 130, 1226-30

Müller-Hülsbeck S (2002): Zerebrale Protektionssysteme für die Behandlung der Karotisstenose: Eine Standortbestimmung. Fortschr Röntgenstr 174, 945-954

Muluk S C, Muluk V S, Sugimoto H, Rhee R Y, Trachtenberg J, Steed D L, Jarrett F, Webster M W, Makaroun M S (1999): Progression of asymptomatic carotid stenosis: a natural history study in 1004 patients. J Vasc Surg 29 (2), 208-14; discussion 214-6

Naghavi M, Libby P, Falk E, Casscells W, Litovsky S, Rumberger J, Badimon J J, Stefanidas C, Morena P, Pasterkamp G et al. (2004): From Vulnerable Plaque to Vulnerable Patient. A Call for New Definitions and Risk Assessment Strategies: Part I. Circulation $\underline{108}, 1664-1672$

Nandalur K R, Baskurt E, Hagspiel K D, Phillips C D, Kramer C M (2005): Calcified carotid atherosclerotic plaque is associated less with ischemic symptoms than is noncalcified plaque on MDCT. AJR Am J Roentgenol 184(1), 295-8

Nandalur K R, Baskurt E, Hagspiel K D, Finch M, Phillips C D, Bollampally S R, Kramer C M (2006): Carotid artery calcification on CT may independently predict stroke risk. AJR Am J Roentgenol 186(2), 547-52

NASCET steering committee (1991): North American Symptomatic Carotid 
Endarterectomy Trial. Methods, patient characteristics, and progress. Stroke $\underline{22}(6)$, $711-20$

Naylor A R, Bolia A, Abbott R J, Pye I F, Smith J, Lennard N, Lloyd A J, London N J, Bell P R (1998): Randomized study of carotid angioplasty and stenting versus carotid endarterectomy: a stopped trial. J Vasc Surg 28(2), 326-34

Ohki T, Parodi J, Veith FJ, Bates M, Bade M, Chang D, Mehta M, Rabin J, Goldstein K, Harvey J, Lipsitz E (2001): Efficacy of a proximal occlusion catheter witch reversal flow in the prevention of embolic events during carotid artery stenting: an eyperimental analysis. J Vasc Surg 33, 504-509

O'Leary D H, Polak J F, Kronmal R A, Kittner S J, Bond M G, Wolfson S K, Jr., Bommer W, Price T R, Gardin J M, Savage P J (1992): Distribution and correlates of sonographically detected carotid artery disease in the Cardiovascular Health Study. The CHS Collaborative Research Group. Stroke 23(12), 1752-60

Park A E, McCarthy W J, Pearce W H, Matsumura J S, Yao J S (1998): Carotid plaque morphology correlates with presenting symptomatology. J Vasc Surg $\underline{27}(5)$, 872-8; discussion 878-9

Parker G J (2004): Analysis of MR diffusion weighted images. Br J Radiol $\underline{77 \text { Spec }}$ No 2 , S176-85

Poeck K, Hacke W: Neurologie: 11.Auflage; Springer Verlag, Berlin-Heidelberg-New York 2001, 185-233

Ranke C, Creutzig A, Becker H, Trappe HJ (1999): Standardization of carotidultrasound: a hemodynamic method normalize for interindividual and interequipment variability. Stroke $\underline{30}, 402-406$

Rantner B, Fraedrich G (2005): Therapiestrategien bei extrakranieller Karotisstenose. Gefäßchirurgie 10, 61-72

Rapp J H, Wakil L, Sawhney R, Pan X M, Yenari M A, Glastonbury C, Coogan S, Wintermark M (2007): Subclinical embolization after carotid artery stenting: new lesions on diffusion-weighted magnetic resonance imaging occur postprocedure. J Vasc Surg 45(5), 867-72; discussion 872-4 
Ravensbergen J, Ravensbergen J W, Krijger J K, Hillen B, Hoogstraten H W (1998): Localizing role of hemodynamics in atherosclerosis in several human vertebrobasilar junction geometries. Arterioscler Thromb Vasc Biol 18(5), 708-16

Reiser H, Kuhn FP, Debus J: Radiologie: 1. Auflage; Thieme Verlag, Stuttgart 2004, 558-612

Riede U N, Werner M, Schaefer HE: Allgemeine und Spezielle Pathologie; 5. Auflage, Georg Thieme Verlag 2004, 422-433

Rothwell P M, Eliasziw M, Gutnikov S A, Fox A J, Taylor D W, Mayberg M R, Warlow C P, Barnett H J (2003): Analysis of pooled data from the randomised controlled trials of endarterectomy for symptomatic carotid stenosis. Lancet 361(9352), 107-16

Saba L, Caddeo G, Sanfilippo R, Montisci R, Mallarini G (2007): Efficacy and sensitivity of axial scans and different reconstruction methods in the study of the ulcerated carotid plaque using multidetector-row CT angiography: comparison with surgical results. AJNR Am J Neuroradiol $\underline{28}(4), 716-23$

Schaar J A, Muller J E, Falk E, Virmani R, Fuster V, Serruys P W, Colombo A, Stefanadis C, Ward Casscells S, Moreno P R et al. (2004): Terminology for high-risk and vulnerable coronary artery plaques. Report of a meeting on the vulnerable plaque, June 17 and 18, 2003, Santorini, Greece. Eur Heart J 로(12), 1077-82

Scheinert D, Schmidt A (2007): Karotisstenose. Bedeutung, Diagnostik, Therapie. Kardiologe 1, 217-228

Schnaudigel S, Groschel K, Pilgram S M, Kastrup A (2008): New brain lesions after carotid stenting versus carotid endarterectomy: a systematic review of the literature. Stroke 39$(6), 1911-9$

Silvennoinen H M, Ikonen S, Soinne L, Railo M, Valanne L (2007): CT angiographic analysis of carotid artery stenosis: comparison of manual assessment, semiautomatic vessel analysis, and digital subtraction angiography. AJNR Am J Neuroradiol $\underline{28}(1)$, 97-103

Stary H C, Chandler A B, Dinsmore R E, Fuster V, Glagov S, Insull W, Jr., Rosenfeld 
M E, Schwartz C J, Wagner W D, Wissler R W (1995): A definition of advanced types of atherosclerotic lesions and a histological classification of atherosclerosis. A report from the Committee on Vascular Lesions of the Council on Arteriosclerosis, American Heart Association. Arterioscler Thromb Vasc Biol 15(9), 1512-31

\section{Statistisches Bundesamt (2006):}

http://www.destatis.de/jetspeed/portal/cms/Sites/destatis/Internet/DE/Content/Statisti ken/Gesundheit/Todesursachen/Tabellen/Content75/GestorbeneAnzahl.psml

Steinberg D, Parthasarathy S, Carew T E, Khoo J C, Witztum J L (1989): Beyond cholesterol. Modifications of low-density lipoprotein that increase its atherogenicity. $\mathrm{N}$ Engl J Med $\underline{320}(14), 915-24$

Stejskal E O, Tanner J E (1965): Spin diffusion measurements: spin echoes in the presence of a time-dependent field gradient. J Chem Phys $\underline{242}$, 288-292

Sterpetti A V, Hunter W J, Schultz R D (1991): Importance of ulceration of carotid plaque in determining symptoms of cerebral ischemia. J Cardiovasc Surg (Torino) $\underline{32}(2), 154-8$

Struffert T, Grunwald I, Roth C, Reith W (2004): Behandlung der arteriosklerotischen Karotisstenose: ein Überblick. Radiologe 44, 936-945

Tedesco M M, Lee J T, Dalman R L, Lane B, Loh C, Haukoos J S, Rapp J H, Coogan S M (2007): Postprocedural microembolic events following carotid surgery and carotid angioplasty and stenting. J Vasc Surg 46⑵, 244-50

Theiss W, Hermanek P, Mathias K, Ahmadi R, Heuser L, Hoffmann F J, Kerner R, Leisch F, Sievert H, von Sommoggy S (2004): Pro-CAS: a prospective registry of carotid angioplasty and stenting. Stroke $\underline{35}(9), 2134-9$

Théron J, Courtheoux P, Alchkar F, Bouvard G, Maiza D (1990): New triple coaxial catheter system of carotid angioplasty with cerebral protection. Am J Neuroradiol 11, 869-874

Thom T, Haase N, Rosamond W, Howard V J, Rumsfeld J, Manolio T, Zheng Z J, Flegal K, O'Donnell C, Kittner S et al. (2006): Heart disease and stroke statistics-2006 update: a report from the American Heart Association Statistics Committee and 
Virmani R, Burke A P, Farb A, Kolodgie F D (2002): Pathology of the unstable plaque. Prog Cardiovasc Dis $\underline{44}(5), 349-56$

Wexler L, Brundage B, Crouse J, Detrano R, Fuster V, Maddahi J, Rumberger J, Stanford W, White R, Taubert K (1996): Coronary artery calcification: pathophysiology, epidemiology, imaging methods, and clinical implications. A statement for health professionals from the American Heart Association. Writing Group. Circulation 94(5), 1175-92

Wholey M H, Al-Mubarek N, Wholey M H (2003): Updated review of the global carotid artery stent registry. Catheter Cardiovasc Interv 60(2), 259-66

Wutke R, Lang W, Fellner C (2002): High-resolution, contrast-enhanced magnetic resonance angiography with elliptical centric k-space ordering of supra-aortic arteries compared with selective X-ray angiography.Stroke $\underline{33}, 1522-9$

Zahn R, Ischinger T, Hochadel M, Zeymer U, Schmalz W, Treese N, Hauptmann K E, Seggewiss H, Janicke I, Haase $H$ et al. (2007): Carotid artery stenting in octogenarians: results from the ALKK Carotid Artery Stent (CAS) Registry. Eur Heart J $\underline{28}(3), 370-5$ 


\section{Lebenslauf}

Ich, Peter Christoph Heinz Albert Weber, wurde am 09.02.1985 in Pfaffenhofen an der IIm geboren. Meine Eltern sind Dr. med. Rainer Weber, Anästhesist an der Ilmtalklinik in Pfaffenhofen an der IIm und Mechthild Weber, geb. Focke, Lehrerin an der Geistschule in Münster. Ich habe drei Geschwister: Philipp Weber, Rechtsreferendar in Hamburg, Christine Weber, Medizinstudentin an der Universität zu Köln und Barbara Weber, Medizinstudentin an der Ludwig-Maximilian-Universität München.

Die Grundschule besuchte ich von 1991 bis 1995 in Kanfen (Frankreich) und von 1995 bis 1998 das Apian-Gymnasium in Ingolstadt. 1998 wechselte ich auf das Annette-von-Droste-Hülshoff-Gymnasium in Münster, an dem ich 2003 mein Abitur ablegte.

Im Juli 2003 trat ich als Soldat auf Zeit in die Luftwaffe der Bundeswehr ein und wechselte kurz darauf als Sanitätsoffiziersanwärter in den Zentralen Sanitätsdienst. Im Mai 2006 wurde ich zum Leutnant ernannt.

Vom Wintersemester 2003/2004 bis Wintersemester 2009/2010 studierte ich Humanmedizin an der Georg-August-Universität in Göttingen. Im August 2005 legte ich den Ersten Abschnitt der Ärztlichen Prüfung und im August 2006, April 2009 und Juli 2009 das United States Medical Licensing Examination (USMLE) für die Approbation in den USA ab. Im November 2009 schloss ich das Studium mit dem Zweiten Abschnitt der Ärztlichen Prüfung ab. Während meines Studiums absolvierte ich u.a. Famulaturen am Bundeswehrkrankenhaus UIm, an der Universität von Accra (Ghana), an der Universität von Kumasi (Ghana) und am NHS District Hospital in York (England). Mein Praktisches Jahr absolvierte ich an der Ammerlandklinik Westerstede, an der Universität von Lausanne (Schweiz), in einer Allgemeinpraxis in Witzenhausen und an der Universität von Louisville (Kentucky, USA).

Neben dem Studium war ich 2008 Intendant des Theaters der StMV Blaue Sänger zu Göttingen. Von 2003 bis 2006 war ich dort als Schauspieler aktiv. Des Weiteren engagiere ich mich politisch zum Thema Entwicklungshilfe und spiele gerne Fußball. 